Article

\title{
Gravitational Waves in Locally Rotationally Symmetric (LRS) Class II Cosmologies
}

\author{
Michael Bradley ${ }^{1, *}$, Mats Forsberg ${ }^{1}$ and Zoltán Keresztes ${ }^{2,3}$ \\ 1 Department of Physics, Umeå University, 90187 Umeå, Sweden; forsberg.mats.a.b@gmail.com \\ 2 Department of Theoretical Physics, University of Szeged, Tisza Lajos krt 84-86, Szeged 6720, Hungary; \\ zkeresztes@titan.physx.u-szeged.hu \\ 3 Department of Experimental Physics, University of Szeged, Dóm Tér 9, Szeged 6720, Hungary \\ * Correspondence: michael.bradley@physics.umu.se
}

Received: 28 August 2017; Accepted: 26 September 2017; Published: 2 October 2017

\begin{abstract}
In this work we consider perturbations of homogeneous and hypersurface orthogonal cosmological backgrounds with local rotational symmetry (LRS), using a method based on the $1+1+2$ covariant split of spacetime. The backgrounds, of LRS class II, are characterised by that the vorticity, the twist of the 2-sheets, and the magnetic part of the Weyl tensor all vanish. They include the flat Friedmann universe as a special case. The matter contents of the perturbed spacetimes are given by vorticity-free perfect fluids, but otherwise the perturbations are arbitrary and describe gravitational, shear, and density waves. All the perturbation variables can be given in terms of the time evolution of a set of six harmonic coefficients. This set decouples into one set of four coefficients with the density perturbations acting as source terms, and another set of two coefficients describing damped source-free gravitational waves with odd parity. We also consider the flat Friedmann universe, which has been considered by several others using the $1+3$ covariant split, as a check of the isotropic limit. In agreement with earlier results we find a second-order wavelike equation for the magnetic part of the Weyl tensor which decouples from the density gradient for the flat Friedmann universes. Assuming vanishing vector perturbations, including the density gradient, we find a similar equation for the electric part of the Weyl tensor, which was previously unnoticed.
\end{abstract}

Keywords: cosmology; covariant split; perturbation theory; gravitational waves

\section{Introduction}

In light of the recent success in measurements of gravitational waves [1], and the consequent opening of a new observational window, it is of interest to study the propagation of gravitational waves and their interactions on different cosmological backgrounds to see, for example, what the effects of anisotropy and/or inhomogeneities are.

The fluctuations in the cosmic microwave background radiation (CMB), the large-scale structures and the cosmological redshift are well explained by the $\Lambda$ CDM model [2-6], which describes an almost homogeneous and isotropic universe with a cosmological constant and cold dark matter. However, there are some deviations between the data and the model. For example, the observed power spectrum of the $\mathrm{CMB}$ seems to differs from the $\Lambda \mathrm{CDM}$ model for large angles [7-10]. Since a large amount of alternative matter is needed to account for the dark sector, a wide range of alternative cosmological models have also been investigated to explore if they can explain the current observations [11-22]. Also, present redshift studies do not give very strict bounds on the anisotropy in the expansion [23-25], making studies of different types of perturbations on anisotropic cosmological backgrounds of interest. For earlier works on this see, for example, [26-31], and for different perturbative methods see [32-36].

In this paper we will use a method based on the $1+3$ and $1+1+2$ covariant splits of spacetime [37-48] to study perturbations on anisotropic backgrounds. In the $1+3$ split there is a preferred timelike 
vector, like the 4-velocity of matter, which is used to split tensors into timelike scalars and spacelike 3 -tensors in a way that keeps covariance of the tensors. Similarly, a further $1+2$ split can be made with respect to a spatial direction. This split is natural when there is a preferred spatial direction on the background, but can be also be used for isotropic backgrounds. Hence, the direction is fixed by, for example, choosing one of the perturbed vectors along it. The gauge problem in relativistic perturbation theory is here avoided by using covariant objects which vanish on the background for the perturbed quantities [49].

In an earlier paper [50] we considered perturbations on a Kantowski-Sachs background, using the $1+1+2$ covariant split of spacetime [45]. The perturbations were vorticity-free and the perturbed spacetime was considered to be described by a perfect fluid. The perturbations include density fluctuations, shear waves, and pure gravitational perturbations travelling with the speed of light at leading order in the high frequency limit. Moreover, beyond this geometrical optics limit, anisotropic dispersion relations were indicated. The full dynamics were found to be given by evolution equations for six harmonic coefficients which decouple into two sub-systems, one with two components describing pure gravitational degrees of freedom, and one with the remaining four coefficients where the density gradient acts as a source term.

In this work we extend the previous $1+1+2$ analysis to a wider class of locally rotationally symmetric (LRS) backgrounds. LRS symmetry means that spacetime is invariant under rotations around at least one spatial direction at every point [51,52]. The analysis will cover vorticity-free perturbations of all LRS spatially homogeneous and hypersurface orthogonal perfect fluid backgrounds with vanishing magnetic part of the Weyl tensor, except the hyperbolic and closed Friedmann models, which together with the flat model have been considered by several authors (see, for example, [37,53-59]), using the $1+3$ split. The considered metrics all belong to LRS class II in the classification of [51]. We find the vorticity-free perturbations of the homogeneous and hypersurface orthogonal LRS II backgrounds to behave in an analogous way to those in the previous study on Kantowski-Sachs backgrounds [50] and a similar harmonic decomposition can be used. Still, all harmonic coefficients can be expressed in terms of six coefficients and the evolution equations have the same structure as before, but the behaviour of the solutions of course varies according to which backgrounds are taken. Finally, as a consistency check, we consider the flat Friedmann background as the isotropic limit of our $1+1+2$ equations. We find that the magnetic part of the Weyl tensor, which in the general anisotropic case is partly sourced by the density gradient, satisfies a source-free second-order damped wave equation. This is in agreement with earlier results, using the $1+3$ covariant split of spacetime. A second-order equation is also obtained for the electric part of the Weyl tensor for the case of pure tensor perturbations, in contrast to an earlier study [53].

The paper is organized as follows: In Section 2 a short summary of the $1+3$ and $1+1+2$ covariant splits of spacetime is given. The LRS backgrounds are discussed in Section 3. In Section 4.1 the harmonic expansion is described and then the evolution equations for the harmonic coefficients are given in Section 4.2. The high-frequency limit is considered in Section 4.3. The flat Friedmann case is treated in Section 4.4. Conclusions are summarised in Section 5.

We use the signature convention $(-+++)$ and units where $c=1$ and $8 \pi G=1$.

\section{The $1+3$ and $1+1+2$ Covariant Splits of Spacetime}

In this section we give a brief summary of the $1+3$ and $1+1+2$ covariant splits of spacetime. For more details on $1+3$ split the reader is referred to [37,42] and for $1+1+2$ split to [45,47]. A summary of the two can also be found in [50].

A $1+3$ split of spacetime is suitable when there is a preferred timelike vector $u^{a}$. The projection operator onto the perpendicular 3-space is given by $h_{a}^{b}=g_{a}^{b}+u_{a} u^{b}$ in terms of the 4-metric $g_{a b}$. 
With the help of $h_{a b}$ vectors and tensors can be covariantly decomposed into spatial and timelike parts. The covariant time derivative and projected spatial derivative are given by

$$
\dot{\psi}_{a . . b} \equiv u^{c} \nabla_{c} \psi_{a \ldots b} \quad \text { and } \quad D_{c} \psi_{a \ldots b} \equiv h_{c}^{f} h_{a}^{d} \ldots h_{b}^{e} \nabla_{f} \psi_{d \ldots e}
$$

respectively. The covariant derivative of the 4-velocity, $u^{a}$, can be decomposed as

$$
\nabla_{a} u_{b}=-u_{a} A_{b}+D_{a} u_{b}=-u_{a} A_{b}+\frac{1}{3} \theta h_{a b}+\omega_{a b}+\sigma_{a b}
$$

where the kinematic quantities of $u^{a}$, acceleration, expansion, vorticity, and shear are defined by $A_{a} \equiv u^{b} \nabla_{b} u_{a}, \theta \equiv D_{a} u^{a}, \omega_{a b} \equiv D_{[a} u_{b]}$, and $\sigma_{a b} \equiv D_{<a} u_{b>}$, respectively. Here square brackets [] denote anti-symmetrisation, and angular brackets $<>$ denote the symmetric and trace-free part of a tensor, i.e. $\psi_{<a b>} \equiv\left(h_{(a}^{c} h_{b)}^{d}-\frac{1}{3} h_{a b} h^{c d}\right) \psi_{c d}$. These quantities, together with the Ricci tensor (expressed via the Einstein equations by, for example, energy density $\mu$ and pressure $p$ for a perfect fluid) and the electric, $E_{a b} \equiv C_{a c b d} u^{c} u^{d}$, and magnetic, $H_{a b} \equiv{ }_{2} \varepsilon_{a d e} C^{d e}{ }_{b c} u^{c}$, parts of the Weyl tensor, are then used as independent variables. Here $\varepsilon_{a b c} \equiv \eta_{d a b c} u^{d} \equiv 4 ! \sqrt{-g} \delta_{[d}^{0} \delta_{a}^{1} \delta_{b}^{2} \delta_{c]}^{3} u^{d}$ is the three-dimensional volume element.

The Ricci identities for $u^{a}$ and the Bianchi identities then provide evolution equations in the $u^{a}$ direction and constraints (see for example [42]).

A formalism for a further split $(1+2)$ with respect to a preferred spatial vector $n^{a}$ (with $u^{a} n_{a}=0$ ) was developed in $[45,47]$. Projections perpendicular to $n^{a}$ are made with $N_{a}^{b}=h_{a}^{b}-n_{a} n^{b}$, and in an analogous way to above, spatial vectors and tensors may be decomposed into scalars along $n^{a}$ and perpendicular two-vectors and symmetric, trace-free two-tensors as $A^{a}=\mathcal{A} n^{a}+\mathcal{A}^{a}, \omega^{a}=\Omega n^{a}+\Omega^{a}$, and $\sigma_{a b}=\Sigma\left(n^{a} n^{b}-\frac{1}{2} N_{a b}\right)+2 \Sigma_{(a} n_{b)}+\Sigma_{a b}$. This occurs similarly for $E_{a b}$ and $H_{a b}$ in terms of $\mathcal{E}, \mathcal{E}_{a}, \mathcal{E}_{a b}$ and $\mathcal{H}, \mathcal{H}_{a}, \mathcal{H}_{a b}$, respectively. Derivatives along and perpendicular to $n^{a}$ are

$$
\widehat{\psi}_{a \ldots b} \equiv n^{c} D_{c} \psi_{a \ldots b}=n^{c} h_{c}^{f} h_{a}^{d} \ldots h_{b}^{e} \nabla_{f} \psi_{d \ldots e} \text { and } \delta_{c} \psi_{a \ldots b} \equiv N_{c}^{f} N_{a}^{d} \ldots N_{b}^{e} D_{f} \psi_{d \ldots e},
$$

respectively. Similarly to the decomposition of $\nabla_{a} u_{b}, D_{a} n_{b}$, and $\dot{n}_{a}$ can be decomposed into further kinematic quantities of $n^{a}$ as

$$
D_{a} n_{b}=n_{a} a_{b}+\frac{1}{2} \phi N_{a b}+\xi \epsilon_{a b}+\zeta_{a b} \quad \text { and } \quad \dot{n}_{a}=\mathcal{A} u_{a}+\alpha_{a}
$$

where $a_{a} \equiv \hat{n}_{a}, \phi \equiv \delta_{a} n^{a}, \xi \equiv \frac{1}{2} \varepsilon^{a b} \delta_{a} n_{b}, \zeta_{a b} \equiv \delta_{\{a} n_{b\}}, \mathcal{A} \equiv n^{a} A_{a}$, and $\alpha_{a} \equiv N_{a}^{b} \dot{n}_{b}$. The two-dimensional volume element is given by $\varepsilon_{a b} \equiv \varepsilon_{a b c} n^{c}$ and curly brackets \{\} denote the symmetric and trace-free part of 2-tensors. A bar on vector indices will denote projection onto the 2-sheets, e.g., $\dot{\psi}_{\bar{a}} \equiv N_{a}^{b} \dot{\psi}_{b}$.

The Ricci and Bianchi identities are then written as constraints and evolution and propagation equations in the $u^{a}$ and $n^{a}$ directions, respectively (see [45]). For the commutation relations between the differential operators ${ }^{\prime}, \widehat{a}$ and $\delta_{a}$ when acting on scalars, vectors and tensors, see Appendix A.

\section{Locally Rotationally Symmetric Spacetimes}

A spacetime which at each point is invariant under rotations around at least one spatial direction is referred to as locally rotationally symmetric, or LRS for short. The corresponding locally maximally symmetric 2-sheets perpendicular to the isotropy axis are characterized by the 2D curvature scalar $\mathcal{R}=2 \mathcal{K} / a_{2}^{2}$, where $a_{2}$ is the radius of curvature (or alternatively the scale factor) and $\mathcal{K}= \pm 1$ or 0 for spheres, pseudo-spheres, or planes, respectively.

The perfect fluid LRS spacetimes can be divided into three classes, I, II and III [51,52]. The Class I metrics are stationary with nonzero vorticity and vanishing shear and expansion and hence are of limited interest as cosmological models. Class II is characterized by the fact that the magnetic Weyl tensor $H_{a b}$, vorticity $\omega_{a b}$, and 2-sheet twisting $\xi$ all vanish. In general, spacetimes in this 
class are both time- and space-dependent and it contains many physically interesting solutions like spherically-symmetric perfect fluids, the inhomogeneous Lemaitre-Tolman-Bondi cosmologies, the homogeneous Kantowski-Sachs and LRS Bianchi I and III cosmologies, and the flat and hyperbolic Friedmann models. The metrics in LRS class III are spatially homogeneous with a nonzero twist of the 2-sheets and have vanishing vorticity and acceleration, as well as vanishing expansion of the 2-sheets. The only models in this class with vanishing magnetic part of the Weyl tensor are the closed Friedmann models.

Perturbations of Kantowski-Sachs universes, which are the hypersurface orthogonal and homogeneous LRS II models with positive 2-curvature, $\mathcal{R}>0$, were studied by us in an earlier paper [50]. In this paper we extend the analysis to all hypersurface orthogonal and homogeneous LRS II models with vanishing expansion of the 2-sheets, i.e., $\phi=0$. With this last requirement a similar harmonic decomposition as in [50] can be used. Fortunately this only excludes the hyperbolic Friedmann universes. Since the only solutions in LRS class III with vanishing magnetic part of the Weyl tensor are the closed Friedmann universes [60], it means that our analysis will cover all homogeneous and hypersurface orthogonal LRS backgrounds with a vanishing magnetic part of the Weyl tensor except the hyperbolic and closed Friedmann models, which together with the flat model have been studied elsewhere with the $1+3$ covariant split (see e.g., [37,53-55,57-59]).

\subsection{LRS Class II}

The perfect fluid LRS Class II spacetimes are characterised by $H_{a b}=\xi=\omega_{a b}=0$, see, e.g., [51,52]. In terms of the quantities defined in Section 2, the spacetimes are given by the following scalars: the energy density $\mu$, the pressure $p$, the electric part of the Weyl tensor $\mathcal{E}$, the expansion $\Theta$, the shear $\Sigma$, the acceleration $\mathcal{A}$, and the expansion of the 2 -sheets, $\phi$. Alternatively, one of the quantities can be replaced with the 2-curvature of the 2 -sheets

$$
\mathcal{R}=\frac{2}{3}(\mu+\Lambda)-2 \mathcal{E}-\frac{1}{2}\left(\Sigma-\frac{2 \Theta}{3}\right)^{2}+\frac{\phi^{2}}{2} .
$$

For a complete local description of the geometry, the frame vectors along the 4-velocity, $u^{a}$, and the preferred spatial direction, $n^{a}$, are needed to construct all Cartan invariants (see e.g., [61]). In terms of timelike and spacelike coordinates, $t$ and $z$, respectively, they are given by

$$
e_{0}=u=X \partial_{t}+x \partial_{z}, \quad e_{1}=n=Y \partial_{t}+y \partial_{z}
$$

where $X, x, Y$ and $y$ are functions of $t$ and $z$. For spatially homogeneous spacetimes, where all invariant objects are functions of a timelike coordinate solely, we may without loss of generality change the time coordinate so that $X=1$ and $x=0$. Metrics with 4 -velocity $u^{a}$ orthogonal to the hypersurfaces of homogeneity are obtained by putting $Y=0$. The assumption of $Y \neq 0$ implies that $\mathcal{R}=0$ (see [52]), and give rise to tilted models of Bianchi types $\mathrm{V}$ or $\mathrm{I}$.

The quantities $\{\mu, p, \mathcal{E}, \Theta, \Sigma, \mathcal{A}, \phi, X, Y, x, y\}$, which describe the spacetime, are subject to integrability conditions given by commutator equations between $e_{0}$ and $e_{1}$, the Ricci equations for $u^{a}$ and $n^{a}$, and some of the Bianchi identities (see [52,61,62]). Einstein's equations are imposed through the Ricci tensor, which for a perfect fluid is given by $\mu, p$ and $u^{a}$.

\subsubsection{Homogeneous and Hypersurface Orthogonal LRS II Metrics}

With the assumptions $x=Y=0$, it follows that the acceleration vanishes, $\mathcal{A}=0$, [52]. The system then reduces to the following evolution equations:

$$
\dot{\mu}=-\Theta(\mu+p),
$$




$$
\begin{gathered}
\dot{\Theta}=-\frac{\Theta^{2}}{3}-\frac{3}{2} \Sigma^{2}-\frac{1}{2}(\mu+3 p)+\Lambda, \\
\dot{\Sigma}=-\left(\frac{2}{3} \Theta+\frac{1}{2} \Sigma\right) \Sigma-\mathcal{E} \\
\dot{\mathcal{E}}=\left(\frac{3}{2} \Sigma-\Theta\right) \mathcal{E}-\frac{1}{2}(\mu+p) \Sigma, \\
\dot{\phi}=-\left(\frac{1}{3} \Theta-\frac{1}{2} \Sigma\right) \phi
\end{gathered}
$$

where a dot indicates derivative with respect to $t$, and to the constraints

$$
\begin{gathered}
\phi \Sigma=\phi \mathcal{E}=0 \\
3 \mathcal{E}=-2(\mu+\Lambda)-3 \Sigma^{2}+\frac{2}{3} \Theta^{2}+\Sigma \Theta-\frac{3}{2} \phi^{2} .
\end{gathered}
$$

3.1.2. Homogeneous and Hypersurface Orthogonal LRS II Metrics with $\phi=0$

For $\phi=0$ the system reduces to

$$
\begin{gathered}
\dot{\mu}=-\Theta(\mu+p), \\
\dot{\Theta}=-\frac{\Theta^{2}}{3}-\frac{3}{2} \Sigma^{2}-\frac{1}{2}(\mu+3 p)+\Lambda, \\
\dot{\Sigma}=\frac{2}{3}(\mu+\Lambda)+\frac{\Sigma^{2}}{2}-\Sigma \Theta-\frac{2}{9} \Theta^{2},
\end{gathered}
$$

with $\mathcal{E}$ given algebraically by

$$
3 \mathcal{E}=-2(\mu+\Lambda)-3 \Sigma^{2}+\frac{2}{3} \Theta^{2}+\Sigma \Theta .
$$

Hence, given an equation of state $p=p(\mu)$ the solutions are completely determined in terms of $\Sigma, \Theta$ and $\mu$, and the nonzero zeroth order quantities are given by the set $S^{(0)}=\{\Sigma, \Theta, \mu, p, \mathcal{E}\}$.

The 2-curvature is now given by

$$
\mathcal{R}=\frac{2}{3}(\mu+\Lambda)-2 \mathcal{E}-\frac{1}{2}\left(\Sigma-\frac{2 \Theta}{3}\right)^{2}=2(\mu+\Lambda)+\frac{3}{2} \Sigma^{2}-\frac{2 \Theta^{2}}{3}=\frac{2 \mathcal{K}}{a_{2}^{2}},
$$

where in the last equality the scale factor of the 2-sheets $a_{2}=a_{2}(t)$ has been introduced and where $\mathcal{K}$ takes the values \pm 1 or 0 according to the geometry of the 2 -sheets: sphere, pseudo-sphere, or flat. Taking the time derivative of $\mathcal{R}$, and using Equations (14)-(16), one finds

$$
\dot{\mathcal{R}}=\left(\Sigma-\frac{2 \Theta}{3}\right) \mathcal{R}
$$

and hence one of the evolution Equations (14)-(16) can be replaced by Equation (19). According to the sign of $\mathcal{R}$, different types of solutions are obtained. For $\mathcal{R}>0$ one gets the Kantowski-Sachs cosmologies, which we studied in [50]. If $\mathcal{R}<0$ the spacetimes are of Bianchi type III. For $\mathcal{R}=0$ there are solutions of Bianchi type $\mathrm{I} \mathrm{VII}_{0}$, including the flat Friedmann universe. Since Equation (18) determines one of the quantities algebraically, one of the evolution equations can be dropped. This is due to the fact that the time derivative of Equation (18) will be identically satisfied due to the evolution Equations (14)-(16).

The line-element can for the different values of $\mathcal{K}$ be written as

$$
d s^{2}=-d t^{2}+a_{1}^{2}(t) d z^{2}+a_{2}^{2}(t)\left(d \vartheta^{2}+f_{\mathcal{K}}(\vartheta) d \varphi^{2}\right)
$$


where $f_{1}(\vartheta)=\sin ^{2} \vartheta, f_{-1}(\vartheta)=\sinh ^{2} \vartheta$, and $f_{0}(\vartheta)=1$ (or alternatively $f_{0}=\vartheta$ ). For $\mathcal{K}=1$ the 2-sheets are spheres and $\vartheta$ and $\varphi$ the usual spherical coordinates, but for $\mathcal{K}=-1$ and 0 the 2-sheets can be taken to be open and infinite with the topology of $R^{2}$. The coordinates are dimensionless and hence the scale factors carry the dimension of length (or time since $c=1$ ). The 4-velocity of comoving observers is $u=\partial / \partial t$ and the direction of anisotropy is $n=a_{1}^{-1} \partial / \partial z$, which due to symmetry and normalisation satisfies [51]:

$$
\hat{n}_{b} \equiv n^{a} D_{a} n_{b}=0, \dot{n}_{\bar{b}}=0 .
$$

In terms of of the scale factors $a_{1}$ and $a_{2}$ in (20), the expansion and scalar part of the shear take the values

$$
\begin{gathered}
\Theta=\frac{\dot{a}_{1}}{a_{1}}+2 \frac{\dot{a}_{2}}{a_{2}}, \\
\Sigma=\frac{2}{3}\left(\frac{\dot{a}_{1}}{a_{1}}-\frac{\dot{a}_{2}}{a_{2}}\right) .
\end{gathered}
$$

\subsubsection{Homogeneous and Hypersurface Orthogonal LRS II Metrics with $\phi \neq 0$}

There are also solutions with $\mathcal{R}=0$ and $\Sigma=\mathcal{E}=0$. For these the sheet expansion $\phi$ is in general nonzero and the system is given by Equations (14) and (15) plus the constraint

$$
\mu+\Lambda-\frac{1}{3} \Theta^{2}+\frac{3}{4} \phi^{2}=0 .
$$

If $\phi \neq 0$, these are the negatively curved Friedmann models of Bianchi type V, whereas $\phi=0$ gives the flat Friedmann models which are covered by the sub-class in Section 3.1.2. For the negatively curved Friedmann models the metric can be given by

$$
d s^{2}=-d t^{2}+a^{2}\left[d \tilde{z}^{2}+e^{-2 z}\left(d x^{2}+d y^{2}\right)\right] .
$$

\section{Vorticity-Free, Perfect Fluid Perturbations of Homogeneous and Orthogonal LRS II Cosmologies}

The analysis of perturbations on Kantowski-Sachs backgrounds in [50] will here be extended to all homogeneous and hypersurface orthogonal LRS class II backgrounds except for the hyperbolic Friedmann models. As for Kantowski-Sachs we will assume that the perturbations are irrotational, i.e., that $\Omega=\Omega^{a}=0$, and also that the perturbed spacetime is described by a perfect fluid. The frame is partly fixed by choosing the preferred timelike vector $u^{a}$ to be the 4-velocity of the fluid also in the perturbed spacetime. Since the preferred direction $n^{a}$ is not kept for the perturbed spacetime, we choose to fix its direction by choosing $a^{a}=0$, meaning that the acceleration only has a component in the $n^{a}$-direction. For more details on the fixing of frame, see [50].

The choice of frame does not completely fix the mapping between the perturbed and background spacetimes [37], but according to the Stewart-Walker lemma [49] variables which vanish on the background are gauge-invariant. Hence, for the generic case with $\phi=0$ on the background, we will replace the nonzero quantities on the background, $\mu, p, \Theta, \Sigma$ and $\mathcal{E}$ with their gradients

$$
\mu_{a}=\delta_{a} \mu, p_{a}=\delta_{a} p, \quad W_{a}=\delta_{a} \Theta, V_{a}=\delta_{a} \Sigma, X_{a}=\delta_{a} \mathcal{E} .
$$

As was shown in [50] the hat derivatives, $\widehat{\Theta}$ etc., are determined in terms of the 2-gradients, $W_{a}$ etc., when the vorticity vanishes (see also Section 4.1.2). The first-order variables which vanish on the background are then

$$
S^{(1)} \equiv\left\{X_{a}, V_{a}, W_{a}, \mu_{a}, p_{a}, \mathcal{A}, \mathcal{A}_{a}, \Sigma_{a}, \Sigma_{a b}, \mathcal{E}_{a}, \mathcal{E}_{a b}, \mathcal{H}, \mathcal{H}_{a}, \mathcal{H}_{a b}, a_{b}, \alpha_{a}, \phi, \xi, \zeta_{a b}\right\} .
$$

These are now subject to the Ricci identities for $u^{a}$ and $n^{a}$ and Bianchi identities, giving evolution equations along $u^{a}$, propagation equations along $n^{a}$ and constraints. The exact, non-perturbative 
system of equations for a $1+1+2$ split of spacetime can be found in [45]. For first-order perturbations the set of equations in terms of the new variables in Equation (26) was derived in [50]. This was done for Kantowski-Sachs backgrounds, but this set is actually valid for all hypersurface orthogonal homogeneous LRS class II spacetimes with $\phi=0$, the differences lying in the zeroth order coefficients for different backgrounds. For completeness we quote the result from [50] in Appendix B.

For the special case $\mathcal{R}=\mathcal{E}=\Sigma=0$ and $\phi \neq 0$ we should in a similar way use the variable $\phi_{a}=\delta_{a} \phi$, whereas $\mathcal{E}$ and $\Sigma$ now are of first order. For the modified system see Appendix $C$.

\subsection{Harmonic Expansion}

For the metrics given by Equation (20), where $\phi=0$, the wave equation on scalars

$$
\nabla^{2} \Psi \equiv g^{a b} \nabla_{a} \nabla_{b} \Psi=0
$$

is separable by applying the following harmonic expansion

$$
\Psi=\sum_{k_{\|}, k_{\perp}} \Psi_{k_{\|} k_{\perp}}^{S} P^{k_{\|}} Q^{k_{\perp}}
$$

where the coefficients $\Psi_{k_{\|} k_{\perp}}^{S}$ depend solely on time (see, e.g., [45,50,63]). The function $P^{k} \|$ is the eigenfunction of the Laplacian $\widehat{\Delta}=n^{a} \nabla_{a} n^{b} \nabla_{b}$ and it is constant on the $z=$ const hypersurfaces

$$
\widehat{\Delta} P^{k_{\|}}=-\frac{k_{\|}^{2}}{a_{1}^{2}} P^{k_{\|}}, \delta_{a} P^{k_{\|}}=\dot{P}^{k_{\|}}=0 .
$$

Here, $k_{\|}$are the dimensionless constant comoving wave numbers in the direction of anisotropy, and $a_{1}$ is the scale factor in this direction. The physical wave numbers are given by $k_{\|} / a_{1}$.

Similarly, harmonics $Q^{k_{\perp}}$ are introduced on the 2-sheets as eigenfunctions to the two-dimensional Laplace-Beltrami operator [45]:

$$
\delta^{2} Q^{k_{\perp}}=-\frac{k_{\perp}^{2}}{a_{2}^{2}} Q^{k_{\perp}}, \widehat{Q}^{k_{\perp}}=\dot{Q}^{k_{\perp}}=0 .
$$

Here, $\delta^{2}=\delta_{a} \delta^{a}, a_{2}$ is the scale factor of the 2-sheets, and $k_{\perp}$ are the dimensionless comoving wavenumbers along the 2-sheets.

When $\mathcal{R}>0$ the 2-sheets are spheres and the harmonics can be represented by the usual spherical harmonics $Y_{l}^{m}$

$$
\delta^{2} Y_{l}^{m}=-\frac{l(l+1)}{a_{2}^{2}} Y_{l}^{m}, \widehat{Y}_{l}^{m}=\dot{Y}_{l}^{m}=0,
$$

with $k_{\perp}^{2}=l(l+1)$. Here $l=0,1,2, \ldots$, and for a given $l$ value the index $m$ runs from $-l$ to $l$. The index $m$ does not occur in the equations governing the perturbations due to the background spacetime symmetries.

For $\mathcal{R} \leq 0$, when the 2 -sheets are open, the $k_{\perp}$ are not discrete and may take any real values. For $\mathcal{R}=0$ the eigenfunctions can be represented by plane waves.

Vectors and tensors can be also expanded in harmonics by introducing vector and tensor harmonics $[47,64,65]$. The even (electric) and odd (magnetic) parity vector harmonics are

$$
Q_{a}^{k_{\perp}}=a_{2} \delta_{a} Q^{k_{\perp}}, \bar{Q}_{a}^{k_{\perp}}=a_{2} \varepsilon_{a b} \delta^{b} Q^{k_{\perp}}
$$


and the vector $\Psi_{a}$ can be expanded as

$$
\Psi_{a}=\sum_{k_{\|}, k_{\perp}} P^{k_{\|}}\left(\Psi_{k_{\|} k_{\perp}}^{V} Q_{a}^{k_{\perp}}+\bar{\Psi}_{k_{\|} k_{\perp}}^{V} \bar{Q}_{a}^{k_{\perp}}\right) .
$$

when the comoving wavenumbers take continous values, the sums are changed to integrals with a convenient normalization factor. For $Q_{a}^{k_{\perp}}$ and $\bar{Q}_{a}^{k_{\perp}}$

$$
\varepsilon^{a b} \delta_{a} Q_{b}^{k_{\perp}}=0 \quad \text { and } \quad \delta^{a} \bar{Q}_{a}^{k_{\perp}}=0
$$

hold, respectively. This corresponds to the fact that a generic vector can be written as the sum of one curl-free and one divergence-free vector.

Similarly, the even and odd tensor harmonics are

$$
Q_{a b}^{k_{\perp}}=a_{2}^{2} \delta_{\{a} \delta_{b\}} Q^{k_{\perp}}, \bar{Q}_{a b}^{k_{\perp}}=a_{2}^{2} \varepsilon_{c\{a} \delta^{c} \delta_{b\}} Q^{k_{\perp}},
$$

and the tensor $\Psi_{a b}$ can be expanded as

$$
\Psi_{a b}=\sum_{k_{\|}, k_{\perp}} P^{k_{\|}}\left(\Psi_{k_{\|} k_{\perp}}^{T} Q_{a b}^{k_{\perp}}+\bar{\Psi}_{k_{\|} k_{\perp}}^{T} \bar{Q}_{a b}^{k_{\perp}}\right) .
$$

Note that for vectors $\Psi_{a}$ and tensors $\Psi_{a b}$ which are odd by definition, the rôles of quantities without and with an overbar, e.g., $\bar{\Psi}_{a}$, are interchanged. For example, for the magnetic part of the Weyl tensor, where the three-dimensional volume element occurs in its definition, $\mathcal{H}_{k_{\|}}^{T} k_{\perp}$ belongs to the odd sector, whereas $\overline{\mathcal{H}}_{k_{\|} k_{\perp}}^{T}$ belongs to the even sector.

Some useful relations involving the vector and tensor harmonics are listed in Appendix D. For different types of harmonics used in relativity and cosmology see, for example, [56,66-68].

\subsubsection{Harmonics When $\phi \neq 0$}

In Bianchi V models with metric

$$
d s^{2}=-d t^{2}+\frac{a^{2}}{z^{2}}\left[d z^{2}+\left(d x^{2}+d y^{2}\right)\right],
$$

where $z$ was introduced as $z=e^{\tilde{z}}$, the source free wave equation for a scalar is:

$$
\begin{aligned}
0 & =\nabla^{2} \Psi=-\ddot{\Psi}-\Theta \Psi+D^{2} \Psi \\
& =-\ddot{\Psi}-\Theta \Psi+\delta^{2} \Psi+\phi \widehat{\Psi}+\widehat{\Delta} \Psi,
\end{aligned}
$$

with

$$
D^{2} \Psi=h^{a b} D_{a} D_{b} \Psi .
$$

In order to separate the time and spatial dependence of $\Psi$, we expand it in harmonics obeying

$$
D^{2} Q^{k}=-\frac{k^{2}}{a^{2}} Q^{k}, \dot{Q}^{k}=0,
$$

where $k$ is a real number. These harmonics can be built as follow. Equation (31) is modified to

$$
\delta^{2} Q^{k_{\perp}}=-k_{\perp}^{2} \frac{z^{2}}{a^{2}} Q^{k_{\perp}}, \widehat{Q}^{k_{\perp}}=\dot{Q}^{k_{\perp}}=0,
$$


where $k_{\perp}$ is real and $Q^{k_{\perp}}$ can be represented by plane waves:

$$
Q^{k_{\perp}}=e^{i k_{\perp}(x+y)} .
$$

The expansion of $Q^{k}$ in harmonics $Q^{k_{\perp}}$ is given by

$$
Q^{k}(x, y, z)=\sum_{k_{\perp}} P^{k, k_{\perp}}(z) Q^{k_{\perp}}(x, y),
$$

where the sum stands for a conveniently normalized integration with respect to $k_{\perp}$ and $P^{k, k_{\perp}}(z)$ satisfies

$$
\left(z^{2} \frac{d^{2}}{d z^{2}}-z-k_{\perp}^{2} z^{2}\right) P^{k, k_{\perp}}(z)=-k^{2} P^{k, k_{\perp}}(z) .
$$

This equation was derived from (41) and (42) and by using $\phi=-2 / a$ [45]. The regular solution of $(45)$ is

$$
P^{k, k_{\perp}}(z)=z^{3 / 2} K_{v}\left(k_{\perp} z\right),
$$

where $K_{v}$ is the modified Bessel functions of the second kind with

$$
v=\sqrt{1-k^{2}} .
$$

A scalar occurring at the first order in the perturbed spacetime can be expanded as

$$
\Psi=\sum_{k} \Psi_{k}^{S}(t) Q^{k}(x, y, z)=\sum_{k, k_{\perp}} \Psi_{k}^{S}(t) P^{k, k_{\perp}}(z) Q^{k_{\perp}}(x, y) .
$$

Nevertheless, this expansion shows that a $1+3$ covariant approach is more convenient in this case than the $1+1+2$. The function $P^{k, k_{\perp}}(z)$ depend on both separation constants $k, k_{\perp}$. This is because $\delta^{2}$-derivative carries a $z^{2}$ factor in Equation (42).

For suitable three-dimensional harmonics see, for example, [38,54-56,68-70] and for $1+3$ analysis of the Friedmann models see, e.g., [37,53-55,57-59].

\subsubsection{Relations between Harmonic Coefficients}

As was shown in [50], on using the commutation relation (A4) and the property (A86) of the vector harmonics and assuming vanishing vorticity, it follows that odd parts of the gradients of the scalars $S^{(0)}=\{\Sigma, \Theta, \mu, p, \mathcal{E}\}$ defined in Equation (26) vanish:

$$
\bar{\mu}_{k_{\|} k_{\perp}}^{V}=\bar{X}_{k_{\|} k_{\perp}}^{V}=\bar{V}_{k_{\|} k_{\perp}}^{V}=\bar{W}_{k_{\|} k_{\perp}}^{V}=\bar{p}_{k_{\|} k_{\perp}}^{V}=0 .
$$

It was also shown that the harmonic coefficients of the hat derivatives of the objects in $S^{(0)}$ can be expressed in terms of the coefficients of the vectors in Equation (26). Denoting an object in $S^{(0)}$ by $G$ its hat derivative can be expanded as

$$
\widehat{G}=\sum_{k_{\|} k_{\perp}} \widetilde{G}_{k_{\|} k_{\perp}} P^{k_{\|}} Q^{k_{\perp}}
$$

and its 2-gradients $G_{a} \equiv \delta_{a} G$ as

$$
G_{a}=\sum_{k_{\|}, k_{\perp}} P^{k_{\|}}\left(G_{k_{\|} k_{\perp}}^{V} Q_{a}^{k_{\perp}}+\bar{G}_{k_{\|} k_{\perp}}^{V} \bar{Q}_{a}^{k_{\perp}}\right) .
$$

From (A3) it then follows that

$$
\widetilde{G}_{k_{\|} k_{\perp}}^{S}=\frac{i k_{\|} a_{2}}{a_{1}} G_{k_{\|} k_{\perp}}^{V}
$$


if $\Omega_{a}$ and $\phi$ vanish to the zeroth order.

\subsection{Evolution Equations for the Case $\phi=0$}

The evolution equations, propagation equations and constraints given in Appendix B can be expanded in harmonics. This will result in time evolution equations and constraints for the harmonic coefficients $\mathcal{A}_{k_{\|} k_{\perp}}^{S}, \mathcal{H}_{k_{\|} k_{\perp}}^{S}, \phi_{k_{\|} k_{\perp}}^{S}, \xi_{k_{\|} k_{\perp}}^{S}, \mu_{k_{\|} k_{\perp}}^{V}, p_{k_{\|} k_{\perp}}^{V}, \mathcal{A}_{k_{\|} k_{\perp}}^{V}, \overline{\mathcal{A}}_{k_{\|} k_{\perp}}^{V}, V_{k_{\|} k_{\perp}}^{V}, W_{k_{\|} k_{\perp}}^{V}, X_{k_{\|} k_{\perp}}^{V}, \Sigma_{k_{\|} k_{\perp}}^{V}, \bar{\Sigma}_{k_{\|} k_{\perp}}^{V}$, $a_{k_{\|} k_{\perp}}^{V}, \bar{a}_{k_{\|} k_{\perp}}^{V}, \alpha_{k_{\|} k_{\perp}}^{V}, \bar{\alpha}_{k_{\|} k_{\perp}}^{V}, \mathcal{E}_{k_{\|} k_{\perp}}^{V}, \overline{\mathcal{E}}_{k_{\|} k_{\perp}}^{V}, \mathcal{H}_{k_{\|} k_{\perp}}^{V}, \overline{\mathcal{H}}_{k_{\|} k_{\perp}}^{V}, \Sigma_{k_{\|} k_{\perp}}^{T}, \bar{\Sigma}_{k_{\|} k_{\perp}}^{T}, \zeta_{k_{\|} k_{\perp}}^{T}, \bar{\zeta}_{k_{\|} k_{\perp}}^{T}, \mathcal{E}_{k_{\|} k_{\perp}}^{T}, \overline{\mathcal{E}}_{k_{\|} k_{\perp}}^{T}, \mathcal{H}_{k_{\|} k_{\perp}}^{T}$, and $\overline{\mathcal{H}}_{k_{\|} k_{\perp}}^{T}$. It follows that $\overline{\mathcal{A}}_{k_{\|} k_{\perp}}^{V}=0$. The frame can then be fixed by requiring $a_{a}=0$, i.e., $a_{k_{\|} k_{\perp}}^{V}=\bar{a}_{k_{\|} k_{\perp}}^{V}=0$ which implies $\tilde{\xi}_{k_{\|} k_{\perp}}^{S}=0$. Finally, by choosing a barytopic equation of state $p=p(\mu)$ we obtain $p_{k_{\|} k_{\perp}}^{V}=c_{s}^{2} \mu_{k_{\|} k_{\perp}}^{V}$ in terms of the speed of sound squared $c_{s}^{2}=d p / d \mu$. Of the remaining 24 harmonic coefficients 18 can be solved for algebraically in terms of the six coefficients $\mu_{k_{\|} k_{\perp}}^{V}, \Sigma_{k_{\|} k_{\perp}}^{T}$, $\mathcal{E}_{k_{\|} k_{\perp}}^{T}, \overline{\mathcal{E}}_{k_{\|} k_{\perp}}^{T}, \mathcal{H}_{k_{\|} k_{\perp}}^{T}$ and $\overline{\mathcal{H}}_{k_{\|} k_{\perp}}^{T}$ (see Appendix E). The remaining system for the six harmonic coefficients decouple into two systems, one for the two coefficients $\overline{\mathcal{E}}_{k_{\|} k_{\perp}}^{T}$ and $\mathcal{H}_{k_{\|} k_{\perp}}^{T}$ and one for the remaining four coefficients $\mu_{k_{\|} k_{\perp}}^{V}, \Sigma_{k_{\|} k_{\perp}}^{T}, \mathcal{E}_{k_{\|} k_{\perp}}^{T}$ and $\overline{\mathcal{H}}_{k_{\|} k_{\perp}}^{T}$

4.2.1. System for $\overline{\mathcal{E}}_{k_{\|} k_{\perp}}^{T}$ and $\mathcal{H}_{k_{\|} k_{\perp}}^{T}$

It turns out that $\overline{\mathcal{E}}_{k_{\|} k_{\perp}}^{T}$ and $\mathcal{H}_{k_{\|} k_{\perp}}^{T}$ decouple from the other coefficients. They satisfy the following system (note that both are of odd parity):

$$
\begin{gathered}
\dot{\mathcal{E}}_{k_{\|} k_{\perp}}^{T}=-\frac{3}{2}(F+\Sigma D) \overline{\mathcal{E}}_{k_{\|} k_{\perp}}^{T}+\frac{i k_{\|}}{a_{1}}(1-D) \mathcal{H}_{k_{\|} k_{\perp}}^{T}, \\
\dot{\mathcal{H}}_{k_{\|} k_{\perp}}^{T}=-\frac{a_{1}}{2 i k_{\|}}\left(\frac{2 k_{\|}^{2}}{a_{1}^{2}}-C B+9 \Sigma E\right) \overline{\mathcal{E}}_{k_{\|} k_{\perp}}^{T}-\frac{3}{2}(2 E+F) \mathcal{H}_{k_{\|} k_{\perp}}^{T},
\end{gathered}
$$

where

$$
\begin{gathered}
B \equiv \frac{2 k_{\|}^{2}}{a_{1}^{2}}+\frac{k_{\perp}^{2}}{a_{2}^{2}}+\frac{9 \Sigma^{2}}{2}+3 \mathcal{E}=\frac{2 k_{\|}^{2}}{a_{1}^{2}}-\frac{\mathcal{R} a_{2}^{2}-k_{\perp}^{2}}{a_{2}^{2}}+3 \Sigma\left(\Sigma+\frac{\Theta}{3}\right), \\
C B \equiv\left(\frac{\mathcal{R} a_{2}^{2}-k_{\perp}^{2}}{a_{2}^{2}}+3 \mathcal{E}\right)=\Sigma\left(\Theta-\frac{3}{2} \Sigma\right)-\frac{k_{\perp}^{2}}{a_{2}^{2}}, \\
D B \equiv \mathcal{R}-\frac{k_{\perp}^{2}}{a_{2}^{2}}+3 \mathcal{E}+\mu+p=\Sigma\left(\Theta-\frac{3}{2} \Sigma\right)-\frac{k_{\perp}^{2}}{a_{2}^{2}}+\mu+p, \\
E B \equiv \frac{\Sigma}{2}(C B-\mathcal{E})+\frac{\Theta \mathcal{E}}{3}=\left(\frac{2}{3} \Theta^{2}-2(\mu+\Lambda)+\Sigma\left(\Theta-\frac{3}{2} \Sigma\right)\right)\left(\Theta-\frac{3}{2} \Sigma\right)-\Sigma \frac{k_{\perp}^{2}}{2 a_{2}^{2}}, \\
F \equiv \Sigma+\frac{2 \Theta}{3} .
\end{gathered}
$$

The system takes the same form as for the Kantowski-Sachs background [50], but note that the functions $B, C$ etc. are slightly differently defined in terms of the curvature $\mathcal{R}$ of the 2-sheets and also that the solutions from Equations (14)-(16), (22) and (23) for the scale factors and kinematic quantities will be different for different values of $\mathcal{R}$, given by Equation (18).

The system can also be written as two decoupled second-order wave equations with damping as

$$
\ddot{\overline{\mathcal{E}}}_{k_{\|} k_{\perp}}^{T}+q_{\overline{\mathcal{E}} 1} \dot{\mathcal{E}}_{k_{\|} k_{\perp}}^{T}+q_{\overline{\mathcal{E}} 0} \overline{\mathcal{E}}_{k_{\|} k_{\perp}}^{T}=0,
$$




$$
\ddot{\mathcal{H}}_{k_{\|} k_{\perp}}^{T}+q_{\mathcal{H} 1} \dot{\mathcal{H}}_{k_{\|} k_{\perp}}^{T}+q_{\mathcal{H} 0} \mathcal{H}_{k_{\|} k_{\perp}}^{T}=0
$$

where

$$
\begin{aligned}
& 2 q_{\overline{\mathcal{E}} 0}=\frac{1-D}{a_{1}}\left[\frac{2 k_{\|}^{2}}{a_{1}}+a_{1}(9 \Sigma E-B C)\right]+3 \frac{d}{d t}(F+\Sigma D) \\
& -3(F+\Sigma D)\left(\frac{d}{d t} \ln \frac{1-D}{a_{1}}-\frac{3}{2}(2 E+F)\right), \\
& q_{\overline{\mathcal{E}} 1}=\frac{3}{2}(2 E+2 F+\Sigma D)-\frac{d}{d t} \ln \frac{1-D}{a_{1}}, \\
& 2 q_{\mathcal{H} 0}=\frac{1-D}{a_{1}}\left[\frac{2 k_{\|}^{2}}{a_{1}}+a_{1}(9 \Sigma E-B C)\right]-3(2 E+F) \frac{d}{d t} \ln \left[\frac{2 k_{\|}^{2}}{a_{1}}+a_{1}(9 \Sigma E-B C)\right] \\
& +\frac{9}{2}(2 E+F)(F+\Sigma D)+3 \frac{d}{d t}(2 E+F) \text {, } \\
& q_{\mathcal{H} 1}=\frac{3}{2}(2 E+2 F+\Sigma D)-\frac{d}{d t} \ln \left[\frac{2 k_{\|}^{2}}{a_{1}}+a_{1}(9 \Sigma E-B C)\right] .
\end{aligned}
$$

In the high-frequency limit the speed of propagation for these waves will approach the speed of light and hence they can be interpreted as free gravitational waves (see Section 4.3).

4.2.2. System for $\Sigma_{k_{\|} k_{\perp}}^{T}, \mathcal{E}_{k_{\|} k_{\perp}}^{T}, \overline{\mathcal{H}}_{k_{\|} k_{\perp}}^{T}$ and $\mu_{k_{\|} k_{\perp}}^{V}$

The coefficients $\Sigma_{k_{\|} k_{\perp}}^{T}, \mathcal{E}_{k_{\|} k_{\perp}}^{T}, \overline{\mathcal{H}}_{k_{\|} k_{\perp}}^{T}$ and $\mu_{k_{\|} k_{\perp}}^{V}$ form the following system:

$$
\begin{aligned}
& \dot{\mu}_{k_{\|} k_{\perp}}^{V}= {\left[\frac{\Sigma}{2}\left(1-3 \frac{\mu+p}{B}\right)-\frac{4 \Theta}{3}\right] \mu_{k_{\|} k_{\perp}}^{V}+\frac{a_{2}}{2}(\mu+p) } \\
& \times\left[(1-C)\left(B \Sigma_{k_{\|} k_{\perp}}^{T}-3 \Sigma \mathcal{E}_{k_{\|} k_{\perp}}^{T}\right)+\frac{i k_{\|}}{a_{1}}(2-J) \overline{\mathcal{H}}_{k_{\|} k_{\perp}}^{T}\right], \\
& \dot{\Sigma}_{k_{\|} k_{\perp}}^{T}=-\frac{c_{s}^{2}}{a_{2}(\mu+p)} \mu_{k_{\|} k_{\perp}}^{V}+\left(\Sigma-\frac{2 \Theta}{3}\right) \Sigma_{k_{\|} k_{\perp}}^{T}-\mathcal{E}_{k_{\|} k_{\perp}}^{T}, \\
& \dot{\mathcal{E}}_{k_{\|} k_{\perp}}^{T}=\frac{3 \Sigma}{2 a_{2} B} \mu_{k_{\|} k_{\perp}}^{V}-\frac{\mu+p}{2} \Sigma_{k_{\|} k_{\perp}}^{T}-\frac{3}{2}(F+\Sigma C) \mathcal{E}_{k_{\|} k_{\perp}}^{T}-\frac{i k_{\|}}{2 a_{1}}(2-J) \overline{\mathcal{H}}_{k_{\|} k_{\perp}}^{T}, \\
& \dot{\mathcal{H}}_{k_{\|} k_{\perp}}^{T}=-\frac{i k_{\|}}{a_{1} a_{2} B} \mu_{k_{\|} k_{\perp}}^{V}-\frac{3}{2}\left(\frac{M}{B}+F\right) \overline{\mathcal{H}}_{k_{\|} k_{\perp}^{T}}-\frac{i k_{\|}}{a_{1}}(1-C) \mathcal{E}_{k_{\|} k_{\perp}}^{T} .
\end{aligned}
$$

Here we have introduced the additional notations

$$
\begin{gathered}
J B \equiv \frac{\left(\mathcal{R} a_{2}^{2}-k_{\perp}^{2}\right) k_{\perp}^{2} a_{1}^{2}}{k_{\|}^{2} a_{2}^{4}}+2 C B=\left(\mathcal{R}-\frac{k_{\perp}^{2}}{a_{2}^{2}}\right)\left(\frac{2 k_{\|}^{2}}{a_{1}^{2}}+\frac{k_{\perp}^{2}}{a_{2}^{2}}\right) \frac{a_{1}^{2}}{k_{\|}^{2}}+6 \mathcal{E} \\
=\frac{k_{\perp}^{2} a_{1}^{2}}{k_{\|}^{2} a_{2}^{2}}\left(\mathcal{R}-\frac{2 k_{\|}^{2}}{a_{1}^{2}}-\frac{k_{\perp}^{2}}{a_{2}^{2}}\right)+2 \Sigma\left(\Theta-\frac{3}{2} \Sigma\right), \\
M \equiv 2 \mathcal{E}\left(\Sigma+\frac{\Theta}{3}\right)+\Sigma \frac{\mathcal{R} a_{2}^{2}-k_{\perp}^{2}}{a_{2}^{2}},
\end{gathered}
$$

where $\mathcal{E}$ is given by Equation (17).

As for the Kantowski-Sachs case, from these one can derive second-order wave-like equations for $\Sigma_{k_{\|} k_{\perp}}^{T}, \mathcal{E}_{k_{\|} k_{\perp}}^{T}$ and $\overline{\mathcal{H}}_{k_{\|} k_{\perp}}^{T}$ where the density gradient $\mu_{k_{\|} k_{\perp}}^{V}$ and its derivative act as source terms. It is only in the high frequency limit that the second-order equations for $\mathcal{E}_{k_{\|} k_{\perp}}^{T}$ and $\overline{\mathcal{H}}_{k_{\|} k_{\perp}}^{T}$ decouple from the source terms, given by the density gradient, and hence describe freely moving gravitational waves. 


\subsection{High-Frequency Approximation}

In [50], where the backgrounds were given by Kantowski-Sachs models, we studied the high-frequency limit (optical limit; see [71,72]), of the propagation equations. For our quantities this implies

$$
\frac{k_{\|}^{2}}{a_{1}^{2}}, \frac{k_{\perp}^{2}}{a_{2}^{2}} \gg \Theta^{2}, \Sigma^{2}, \mathcal{E}, \mu, p .
$$

Since in this limit the curvature of the 2-sheets becomes negligible the resulting equations are identical in form to those for the Kantowski-Sachs backgrounds for all signs of $\mathcal{R}$, but the zeroth-order factors $a_{1}, a_{2}, \Theta$ and $\Sigma$ of course are different for different backgrounds.

For the uncoupled system of $\overline{\mathcal{E}}_{k_{\|} k_{\perp}}^{T}$ and $\mathcal{H}_{k_{\|} k_{\perp}}^{T}$ the following second-order wave equations with damping

$$
\begin{aligned}
& \ddot{\mathcal{E}}_{k_{\|} k_{\perp}}^{T}+q_{\overline{\mathcal{E}} 1} \dot{\overline{\mathcal{E}}}_{k_{\|} k_{\perp}}^{T}+\left(\frac{k_{\|}^{2}}{a_{1}^{2}}+\frac{k_{\perp}^{2}}{a_{2}^{2}}\right) \overline{\mathcal{E}}_{k_{\|} k_{\perp}}^{T}=0, \\
& \ddot{\mathcal{H}}_{k_{\|} k_{\perp}}^{T}+q_{\mathcal{H} 1} \dot{\mathcal{H}}_{k_{\|} k_{\perp}}^{T}+\left(\frac{k_{\|}^{2}}{a_{1}^{2}}+\frac{k_{\perp}^{2}}{a_{2}^{2}}\right) \mathcal{H}_{k_{\|} k_{\perp}}^{T}=0,
\end{aligned}
$$

where

$$
\begin{gathered}
q_{\overline{\mathcal{E}}_{1}}=\frac{7 \Theta}{3}+4 \Sigma-3 \Sigma \frac{k_{\perp}^{2}}{\frac{a_{2}^{2}}{a_{1}^{2}} k_{\|}^{2}+k_{\perp}^{2}}, \\
q_{\mathcal{H}_{1}}=\frac{7 \Theta}{3}+4 \Sigma-6 \Sigma \frac{k_{\perp}^{2}}{\frac{2 a_{2}^{2}}{a_{1}^{2}} k_{\|}^{2}+k_{\perp}^{2}},
\end{gathered}
$$

are obtained. These are in the form

$$
\ddot{X}+2 \zeta \Omega \dot{X}+\Omega^{2} X=0,
$$

where $\Omega$ is the undamped angular frequency and the actual angular frequency is given by $\Omega \sqrt{1-\zeta^{2}}$. The propagation speed of the wave is

$$
c_{w}=\frac{\Omega}{k_{\text {phys }}} \sqrt{1-\zeta^{2}} \quad \text { with } \quad k_{\text {phys }}^{2}=\frac{k_{\|}^{2}}{a_{1}^{2}}+\frac{k_{\perp}^{2}}{a_{2}^{2}} .
$$

The propagation velocity hence goes as $1-\zeta^{2} / 2$ for relatively small damping coefficients $\zeta$, and approaches the speed of light for large frequencies. For the static case, when $\Theta=\Sigma=0$, the damping would vanish and then the propagation velocity would be exactly the speed of light. When the propagation is along the preferred direction, $k_{\perp}=0$, and $q_{\overline{\mathcal{E}}_{1}}=q_{\mathcal{H}_{1}}$, resulting in the same damping $\zeta_{\|}$for both variables $\overline{\mathcal{E}}_{k_{\|} k_{\perp}}^{T}$ and $\mathcal{H}_{k_{\|} k_{\perp}}^{T}$. Hence, they have the same propagation velocity which differs from the speed of light at the second-order in $\zeta_{\|}$. However, when the propagation is perpendicular to the preferred direction, $k_{\|}^{2}=0$, then $q_{\overline{\mathcal{E}}_{1}} \neq q_{\mathcal{H}_{1}}$, giving different dampings $\zeta_{\perp \overline{\mathcal{E}}}$ and $\zeta_{\perp \mathcal{H}}$ for $\overline{\mathcal{E}}_{k_{\|} k_{\perp}}^{T}$ and $\mathcal{H}_{k_{\|} k_{\perp}}^{T}$, respectively. Therefore, the propagation velocities also differ at the second order in damping coefficients. In addition, since $\zeta_{\|} \neq \zeta_{\perp \overline{\mathcal{E}}} \neq \zeta_{\perp \mathcal{H}}$, the propagation velocities are direction-dependent.

As mentioned in Section 4.2.2, the second-order equations for $\mathcal{E}_{k_{\|} k_{\perp}}^{T}$ and $\overline{\mathcal{H}}_{k_{\|} k_{\perp}}^{T}$ also decouple from the density gradient in the high frequency limit and are given by

$$
\ddot{\mathcal{E}}_{k_{\|} k_{\perp}}^{T}+q_{\mathcal{E} 1} \dot{\mathcal{E}}_{k_{\|} k_{\perp}}^{T}+\left(\frac{k_{\|}^{2}}{a_{1}^{2}}+\frac{k_{\perp}^{2}}{a_{2}^{2}}\right) \mathcal{E}_{k_{\|} k_{\perp}}^{T}=0,
$$




$$
\ddot{\overline{\mathcal{H}}}_{k_{\|} k_{\perp}}^{T}+q_{\overline{\mathcal{H}} 1} \dot{\mathcal{H}}_{k_{\|} k_{\perp}}^{T}+\left(\frac{k_{\|}^{2}}{a_{1}^{2}}+\frac{k_{\perp}^{2}}{a_{2}^{2}}\right) \mathcal{H}_{k_{\|} k_{\perp}}^{T}=0
$$

with $q_{\mathcal{E} 1}=q_{\mathcal{H}_{1}}$ and $q_{\overline{\mathcal{H}} 1}=q_{\overline{\mathcal{E}}_{1}}$. Beause of the latter, the propagation velocities of $\mathcal{E}_{k_{\|} k_{\perp}}^{T}$ and $\mathcal{H}_{k_{\|}}^{T} k_{\perp}$ and of $\overline{\mathcal{H}}_{k_{\|} k_{\perp}}^{T}$ and $\overline{\mathcal{E}}_{k_{\|} k_{\perp}}^{T}$ coincide in the high-frequency limit and as before the propagation velocity differs from the speed of light to the second order in the damping parameter.

\subsection{Perturbations of the flat Friedmann models}

Here we consider the flat Friedmann models as a check of the isotropic limit of the general LRS II case. In this case there is no preferred spatial direction on the background, but as before, we fix the 1-direction by choosing the acceleration to only have a 1-component, i.e., $a_{a}=0$, in the perturbed spacetime. Tensor perturbations of the Friedmann cases, using the $1+3$ covariant split, were studied in [53] and we make a comparison with their results.

The flat Friedmann models are given by $\mathcal{E}=\Sigma=\mathcal{R}=\phi=0$. Without loss of generality we can use $a_{1}=a_{2} \equiv a$. We also introduce the notations

$$
k^{2} \equiv k_{\|}^{2}+k_{\perp}^{2}
$$

and

$$
B_{0}=\frac{2 k_{\|}^{2}}{a^{2}}+\frac{k_{\perp}^{2}}{a^{2}}
$$

The first system for $\overline{\mathcal{E}}_{k_{\|} k_{\perp}}^{T}$ and $\mathcal{H}_{k_{\|} k_{\perp}}^{T}$ then reduces to

$$
\begin{gathered}
\dot{\mathcal{E}}_{k_{\|} k_{\perp}}^{T}=-\Theta \overline{\mathcal{E}}_{k_{\|} k_{\perp}}^{T}+\frac{i k_{\|}}{a B_{0}}\left(\frac{2 k^{2}}{a^{2}}-\mu-p\right) \mathcal{H}_{k_{\|} k_{\perp}}^{T} \\
\dot{\mathcal{H}}_{k_{\|} k_{\perp}}^{T}=-\frac{a}{2 i k_{\|}} B_{0} \overline{\mathcal{E}}_{k_{\|} k_{\perp}}^{T}-\Theta \mathcal{H}_{k_{\|} k_{\perp}}^{T} .
\end{gathered}
$$

As before, this can be written as second-order damped wave equations

$$
\ddot{\overline{\mathcal{E}}}_{k_{\|} k_{\perp}}^{T}+\left(\frac{7 \Theta}{3}-N\right) \dot{\overline{\mathcal{E}}}_{k_{\|} k_{\perp}}^{T}+\left(\frac{k^{2}}{a^{2}}+\frac{2}{3} \Theta^{2}+2(\Lambda-p)-N \Theta\right) \overline{\mathcal{E}}_{k_{\|} k_{\perp}}^{T}=0
$$

and

$$
\ddot{\mathcal{H}}_{k_{\|} k_{\perp}}^{T}+\frac{7}{3} \Theta \dot{\mathcal{H}}_{k_{\|} k_{\perp}}^{T}+\left(\frac{k^{2}}{a^{2}}+\frac{2}{3} \Theta^{2}+2(\Lambda-p)\right) \mathcal{H}_{k_{\|} k_{\perp}}^{T}=0 .
$$

The term $N$ is given by

$$
N \equiv \frac{(\mu+p)\left(1+3 c_{s}^{2}\right)}{3\left(\frac{2 k^{2}}{a^{2}}-(\mu+p)\right)} \Theta
$$

and vanishes in the high frequency limit.

The second system becomes

$$
\begin{gathered}
\dot{\mu}_{k_{\|} k_{\perp}}^{V}=-\frac{4 \Theta}{3} \mu_{k_{\|} k_{\perp}}^{V}+\frac{k^{2}}{a}(\mu+p) \Sigma_{k_{\|} k_{\perp}}^{T}-\frac{a^{2}(\mu+p) B_{0}}{2 i k_{\|}} \overline{\mathcal{H}}_{k_{\|} k_{\perp}}^{T}, \\
\dot{\Sigma}_{k_{\|} k_{\perp}}^{T}=-\frac{c_{S}^{2}}{a(\mu+p)} \mu_{k_{\|} k_{\perp}}^{V}-\frac{2 \Theta}{3} \Sigma_{k_{\|} k_{\perp}}^{T}-\mathcal{E}_{k_{\|} k_{\perp}}^{T}, \\
\dot{\mathcal{E}}_{k_{\|} k_{\perp}}^{T}=-\frac{\mu+p}{2} \Sigma_{k_{\|} k_{\perp}}^{T}-\Theta \mathcal{E}_{k_{\|} k_{\perp}}^{T}+\frac{a B_{0}}{2 i k_{\|}} \overline{\mathcal{H}}_{k_{\|} k_{\perp}}^{T},
\end{gathered}
$$




$$
\dot{\mathcal{\mathcal { H }}}_{k_{\|} k_{\perp}}^{T}=-\frac{i k_{\|}}{a^{2} B_{0}} \mu_{k_{\|} k_{\perp}}^{V}-\Theta \overline{\mathcal{H}}_{k_{\|} k_{\perp}}^{T}-\frac{2 i k_{\|} k^{2}}{a^{3} B_{0}} \mathcal{E}_{k_{\|} k_{\perp}}^{T} .
$$

From these we obtain the following second-order wave equations for $\Sigma_{k_{\|} k_{\perp}}^{T}$ and $\mathcal{E}_{k_{\|} k_{\perp}}^{T}$, again with the density fluctuations acting as source terms,

$$
\begin{gathered}
\ddot{\Sigma}_{k_{\|} k_{\perp}}^{T}+\frac{5 \Theta}{3} \dot{\Sigma}_{k_{\|} k_{\perp}}^{T}+\left(\frac{k^{2}}{a^{2}}+\frac{\Theta^{2}}{6}+\frac{3}{2}(\Lambda-p)\right) \Sigma_{k_{\|} k_{\perp}}^{T}=\frac{\left(1-c_{s}^{2}\right)}{a(\mu+p)} \dot{\mu}_{k_{\|} k_{\perp}}^{V}+s_{\Sigma 0} \mu_{k_{\|} k_{\perp}}^{V}, \\
\ddot{\mathcal{E}}_{k_{\|} k_{\perp}}^{T}+\left(\frac{7 \Theta}{3}-N\right) \dot{\mathcal{E}}_{k_{\|} k_{\perp}}^{T}+\left(\frac{k^{2}}{a^{2}}+\frac{2}{3} \Theta^{2}+2(\Lambda-p)-N \Theta\right) \mathcal{E}_{k_{\|} k_{\perp}}^{T}=\frac{N}{a(\mu+p)} \dot{\mu}_{k_{\|} k_{\perp}}^{V}+s_{\mathcal{E} 0} \mu_{k_{\|} k_{\perp}}^{V},
\end{gathered}
$$

where

$$
s_{\Sigma 0}=\frac{\left(\left(\frac{4}{3}-\frac{5}{3} c_{s}^{2}-c_{s}^{4}\right) \Theta-2 c_{s} \dot{c}_{s}\right)}{a(\mu+p)} \text { and } \quad s_{\mathcal{E} 0}=\frac{c_{s}^{2}-1}{2 a}+\frac{4 \Theta N}{3 a(\mu+p)} .
$$

The density gradient obeys the following second-order equation

$$
\ddot{\mu}_{k_{\|} k_{\perp}}^{V}+\left(\frac{10}{3}+c_{s}^{2}\right) \theta \dot{\mu}_{k_{\|} k_{\perp}}^{V}+\left(\frac{c_{s}^{2} k^{2}}{a^{2}}+\frac{11}{6} \theta^{2}+\frac{4 c_{s}^{2}}{3} \theta^{2}-\frac{5}{2}(p-\Lambda)\right) \mu_{k_{\|} k_{\perp}}^{V}=0
$$

and we see that the density perturbations propagate with the speed of sound in the high frequency limit. Unlike the case with an anisotropic background, we now obtain a second-order decoupled wave equation for $\overline{\mathcal{H}}_{k_{\|} k_{\perp}}^{T}$

$$
\ddot{\mathcal{H}}_{k_{\|} k_{\perp}}^{T}+\frac{7}{3} \Theta \dot{\overline{\mathcal{H}}}_{k_{\|} k_{\perp}}^{T}+\left(\frac{k^{2}}{a^{2}}+\frac{2}{3} \Theta^{2}+2(\Lambda-p)\right) \overline{\mathcal{H}}_{k_{\|} k_{\perp}}^{T}=0 .
$$

Note that Equations (86) and (96) for $\mathcal{H}_{k_{\|} k_{\perp}}^{T}$ and $\overline{\mathcal{H}}_{k_{\|} k_{\perp}}^{T}$, respectively, are identical. Similarly, the left-hand sides of Equations (85) for $\overline{\mathcal{E}}_{k_{\|} k_{\perp}}^{T}$ and (93) for $\mathcal{E}_{k_{\|} k_{\perp}}^{T}$, respectively, are identical. The damping coefficients in the second-order equations depend only on $k$, therefore the propagation velocities of the perturbations are not direction-dependent. In addition the propagation velocities at high frequencies approach the speed of light. Because of the symmetries of Friedmann spacetimes, we can safely assume that $\mu_{k_{\|} k_{\perp}}^{V}$ depend only on $k$, and not separately on $k_{\|}$and $k_{\perp}$, which is also clear from Equation (95). Then the second-order equations for $\Sigma_{k_{\|} k_{\perp}}^{T}$ and $\mathcal{E}_{k_{\|} k_{\perp}}^{T}$ also depend only on $k$ like those governing $\overline{\mathcal{E}}_{k_{\|} k_{\perp}}^{T}$, $\mathcal{H}_{k_{\|} k_{\perp}}^{T}$ and $\overline{\mathcal{H}}_{k_{\|} k_{\perp}}^{T}$.

In [53] the $1+3$ covariant split was used to study tensorial perturbations of the Friedmann models. The pure tensor perturbations are characterised by vanishing energy density gradients and vorticity to the first order. Hence, when comparing our result with their we must use $\mu_{k_{\|} k_{\perp}}^{V}=\dot{\mu}_{k_{\|} k_{\perp}}^{V}=0$. Our Equations (86) and (96) for the even and odd parts of the magnetic part of the Weyl tensor, $\overline{\mathcal{H}}_{k_{\|} k_{\perp}}^{T}$ and $\mathcal{H}_{k_{\|} k_{\perp}}^{T}$, respectively, are then the same as their Equation (20) for the flat Friedmann case. The equation for $\Sigma_{k_{\|} k_{\perp}}^{T}$ (92) similarly corresponds to their Equation (22) ${ }^{1}$.

The main difference between [53] and our result is that we obtain a second-order wave equation from Equation (93) for $\mathcal{E}_{k_{\|} k_{\perp}}^{T}$, whereas they needed a third-order equation to decouple $E_{a b}$. In Equation (15) in [53] they give a second-order equation for the electric part of the Weyl tensor,

1 There seems to be a misprint in Equation (22) it [53]. Probably the factor $(9 \gamma-1)$ should read $(10-9 \gamma)$. 
$E_{a b}$, with the shear $\sigma_{a b}$ acting as a source term. From our Equation (88) we see that imposing $\mu_{k_{\|} k_{\perp}}^{V}=\dot{\mu}_{k_{\|} k_{\perp}}^{V}=0$ gives a constraint between $\Sigma_{k_{\|} k_{\perp}}^{T}$ and $\overline{\mathcal{H}}_{k_{\|} k_{\perp}}^{T}$

$$
\Sigma_{k_{\|} k_{\perp}}^{T}=\frac{a^{3} B_{0}}{2 k^{2} i k_{\|}} \overline{\mathcal{H}}_{k_{\|} k_{\perp}}^{T}
$$

This constraint is satisfied as is seen by differentiating it and substituting Equations (89)-(91). Hence, the number of independent variables in the system (88)-(91) is reduced from four to two by imposing that the density perturbations vanish. With the help of Equation (90) now $\Sigma_{k_{\|} k_{\perp}}^{T}$ can be completely expressed in terms of $\mathcal{E}_{k_{\|} k_{\perp}}^{T}$ and $\dot{\mathcal{E}}_{k_{\|}}^{T} k_{\perp}$

$$
\Sigma_{k_{\|} k_{\perp}}^{T}=\frac{2 a^{2}}{2 k^{2}-(\mu+p) a^{2}}\left(\dot{\mathcal{E}}_{k_{\|} k_{\perp}}^{T}+\Theta \mathcal{E}_{k_{\|} k_{\perp}}^{T}\right)
$$

By using this result in their Equation (15) in for the flat Friedmann case, where simple plane waves can be used for the harmonics, we obtain our Equation (93). The three-dimensional Equations (7) and (21) in [53] correspond to our Equations (97) and (98). Using the three-dimensional harmonics in [68] similar second-order equations for all values of $\mathcal{K}$ are obtained (see Appendix F).

\section{Conclusions}

A previous analysis of vorticity-free perturbations on Kantowski-Sachs backgrounds has been extended to all homogeneous and hypersurface orthogonal LRS perfect fluids with the vanishing magnetic part of the Weyl tensor except the hyperbolic and closed Friedmann models, which have been studied elsewere [37,53-55,57-59] using the covariant $1+3$ split approach. We find the same structure of the evolution equations for the perturbations as in the case of Kantowski-Sachs. All harmonic coefficients can be determined in terms of a subset containing only six coefficients. The evolution equations for these decouple into one system for $\overline{\mathcal{E}}_{k_{\|} k_{\perp}}^{T}$ and $\mathcal{H}_{k_{\|} k_{\perp}}^{T}$, representing source-free gravitational degrees of freedom, and another for $\Sigma_{k_{\|} k_{\perp}}^{T}, \mathcal{E}_{k_{\|} k_{\perp}}^{T}, \overline{\mathcal{H}}_{k_{\|} k_{\perp}}^{T}$, and $\mu_{k_{\|} k_{\perp}}^{V}$, which describes perturbations sourced by the density gradient. Only in the high frequency limit do the second-order wave equations for $\mathcal{E}_{k_{\|}}^{T} k_{\perp}$ and $\overline{\mathcal{H}}_{k_{\|}}^{T} k_{\perp}$ decouple from the source terms. The analysis of propagation velocities in the high frequency limit led to direction-dependent dispersion relations on anisotropic backgrounds.

We also studied perturbations on the flat Friedmann universe, which is the isotropic limit of the considered class of backgrounds. Here the second-order wave equation for $\overline{\mathcal{H}}_{k_{\|} k_{\perp}}^{T}$ decouples from the other coefficients, whereas $\mathcal{E}_{k_{\|} k_{\perp}}^{T}$ still is sourced by the density gradient. The result is compared with an earlier study, [53], where a $1+3$ formalism was used to study pure tensor perturbations on Friedmann backgrounds. The $1+1+2$ covariant approach, together with a harmonic decomposition, is also effective for this setup, because all tensorial perturbations are easily obtained as second-order differential equations in contrast to the third-order equation more naturally appearing for the electric part of Weyl tensor in the $1+3$ covariant description.

Acknowledgments: The work of ZK was partially supported by the UNKP-17-4 New National Excellence Program of the Ministry of Human Capacities and partially by the Hungarian National Research, Development and Innovation Office (NKFI) in the form of the grant 123996.

Author Contributions: All the authors contributed equally to the paper.

Conflicts of Interest: The authors declare no conflict of interest. 


\section{Appendix A. Commutation Relations}

The commutation relations of covariant derivatives of the scalar field $\Psi$ on hypersurface orthogonal and homogeneous LRS II backgrounds are to the first order:

$$
\begin{gathered}
\hat{\dot{\Psi}}-\dot{\hat{\Psi}}=-\mathcal{A} \dot{\Psi}+\left(\Sigma+\frac{\Theta}{3}\right) \widehat{\Psi}, \\
\delta_{a} \dot{\Psi}-N_{a}^{b}\left(\delta_{b} \Psi\right)^{\cdot}=-\mathcal{A}_{a} \dot{\Psi}-\frac{1}{2}\left(\Sigma-\frac{2 \Theta}{3}\right) \delta_{a} \Psi, \\
\delta_{a} \widehat{\Psi}-N_{a}{ }^{b}\left(\widehat{\delta_{b} \Psi}\right)=-2 \varepsilon_{a b} \Omega^{b} \dot{\Psi}+\frac{1}{2} \phi \delta_{a} \Psi, \\
\delta_{[a} \delta_{b]} \Psi=\varepsilon_{a b} \Omega \dot{\Psi} .
\end{gathered}
$$

Similar relations hold for the first-order 2-vector $\Psi_{a}$ :

$$
\begin{gathered}
\hat{\dot{\Psi}}_{\bar{a}}-\dot{\hat{\Psi}}_{\bar{a}}=\left(\Sigma+\frac{\Theta}{3}\right) \widehat{\Psi}_{\bar{a}}, \\
\delta_{a} \dot{\Psi}_{b}-N_{a}{ }^{c} N_{b}{ }^{d}\left(\delta_{c} \Psi_{d}\right)=-\frac{1}{2}\left(\Sigma-\frac{2 \Theta}{3}\right) \delta_{a} \Psi_{b}, \\
\delta_{a} \widehat{\Psi}_{b}-N_{a}{ }^{c} N_{b}{ }^{d}\left(\widehat{\delta}_{c} \Psi_{d}\right)=\frac{1}{2} \phi \delta_{a} \Psi_{b}, \\
\delta_{[a} \delta_{b]} \Psi_{c}=\frac{1}{2} \mathcal{R} N_{c[a} \Psi_{b]},
\end{gathered}
$$

and for the first-order symmetric, trace-free 2-tensor $\Psi_{a b}$ :

$$
\begin{gathered}
\widehat{\hat{\Psi}}_{\{a b\}}-\dot{\hat{\Psi}}_{\{a b\}}=\left(\Sigma+\frac{\Theta}{3}\right) \widehat{\Psi}_{\bar{a}}, \\
\delta_{a} \dot{\Psi}_{b c}-N_{a}{ }^{d}{N_{b}}^{e} N_{c}{ }^{f}\left(\delta_{d} \Psi_{e f}\right)=-\frac{1}{2}\left(\Sigma-\frac{2 \Theta}{3}\right) \delta_{a} \Psi_{b c}, \\
\delta_{a} \widehat{\Psi}_{b c}-N_{a}{ }^{d} N_{b}{ }^{e} N_{c}{ }^{f}\left(\delta_{d} \widehat{\Psi}_{e f}\right)=0, \\
2 \delta_{[a} \delta_{b]} \Psi_{c d}=\mathcal{R}\left(N_{c[a} \Psi_{b] d}+N_{d[a} \Psi_{b] c}\right),
\end{gathered}
$$

where the 2-curvature $\mathcal{R}$ is given by Equation (18). In (A3) and (A7) the last terms on the right-hand sides are second-order in the generic case when $\phi=0$ on the background, and can hence be dropped.

\section{Appendix B. Evolution and Propagation Equations and Constraints for $\phi=0$}

Here, the evolution and propagation equations and constraints from reference [50] for the generic case when $\phi=0$ are repeated. These are obtained by linearising the $1+1+2$ equations in [45]. ${ }^{2}$

The set $S^{(0)}=\{\Theta, \Sigma, \mathcal{E}, \mu, p\}$ gives the quantities which are nonzero on the background. We define the corresponding first-order quantities

$$
\mu_{a}=\delta_{a} \mu, p_{a}=\delta_{a} p, \quad W_{a}=\delta_{a} \Theta, V_{a}=\delta_{a} \Sigma, X_{a}=\delta_{a} \mathcal{E} .
$$

2 There are some minor misprints in [45]. See [50] for corrections. 
which vanish on the background. The vorticity-free perturbations are then given by the following nonzero first-order quantities (which all vanish on the background):

$$
S^{(1)} \equiv\left\{X_{a}, V_{a}, W_{a}, \mu_{a}, p_{a}, \mathcal{A}, \mathcal{A}_{a}, \Sigma_{a}, \Sigma_{a b}, \mathcal{E}_{a}, \mathcal{E}_{a b}, \mathcal{H}, \mathcal{H}_{a}, \mathcal{H}_{a b}, a_{b}, \alpha_{a}, \phi, \xi, \zeta_{a b}\right\}
$$

The evolution equations are given by

$$
\begin{aligned}
& \dot{\phi}=\left(\Sigma-\frac{2 \Theta}{3}\right)\left(\frac{\phi}{2}-\mathcal{A}\right)+\delta^{a} \alpha_{a}, \\
& 2 \dot{\xi}=\left(\Sigma-\frac{2 \Theta}{3}\right) \xi+\varepsilon^{a b} \delta_{a} \alpha_{b}+\mathcal{H}, \\
& \dot{\mathcal{H}}=\frac{3}{2}\left(\Sigma-\frac{2 \Theta}{3}\right) \mathcal{H}-\varepsilon^{a b} \delta_{a} \mathcal{E}_{b}-3 \mathcal{E} \xi, \\
& \dot{\mu}_{\bar{a}}=\frac{1}{2}\left(\Sigma-\frac{2 \Theta}{3}\right) \mu_{a}-\Theta\left(\mu_{a}+p_{a}\right)-(\mu+p) W_{a}+\dot{\mu} \mathcal{A}_{a}, \\
& \dot{X}_{\bar{a}}=2\left(\Sigma-\frac{2 \Theta}{3}\right) X_{a}+\frac{3 \mathcal{E}}{2}\left(V_{a}-\frac{2}{3} W_{a}\right)-\frac{\mu+p}{2} V_{a}-\frac{\Sigma}{2}\left(\mu_{a}+p_{a}\right)+\dot{\mathcal{E}} \mathcal{A}_{a}+\varepsilon^{b c} \delta_{a} \delta_{b} \mathcal{H}_{c} ， \\
& \dot{V}_{\bar{a}}-\frac{2}{3} \dot{W}_{\bar{a}}=\frac{3}{2}\left(\Sigma-\frac{2 \Theta}{3}\right)\left(V_{a}-\frac{2}{3} W_{a}\right)-X_{a}+\frac{1}{3}\left(\mu_{a}+3 p_{a}\right)+\left(\dot{\Sigma}-\frac{2 \dot{\Theta}}{3}\right) \mathcal{A}_{a}-\delta_{a} \delta^{b} \mathcal{A}_{b}, \\
& \dot{\Sigma}_{\{a b\}}=\left(\Sigma-\frac{2 \Theta}{3}\right) \Sigma_{a b}+\delta_{\{a} \mathcal{A}_{b\}}-\mathcal{E}_{a b}, \\
& \dot{\zeta}_{\{a b\}}=\frac{1}{2}\left(\Sigma-\frac{2 \Theta}{3}\right) \zeta_{a b}+\delta_{\{a} \alpha_{b\}}-\varepsilon_{c\{a} \mathcal{H}_{b\}}^{c} .
\end{aligned}
$$

The equations containing both propagation and evolution contributions are

$$
\begin{gathered}
\dot{W}_{\bar{a}}-\delta_{a} \widehat{\mathcal{A}}=\left(\frac{\Sigma}{2}-\Theta\right) W_{a}-3 \Sigma V_{a}-\frac{1}{2}\left(\mu_{a}+3 p_{a}\right)+\dot{\Theta} \mathcal{A}_{a}+\delta_{a} \delta^{b} \mathcal{A}_{b}, \\
\widehat{\alpha}_{\bar{a}}-\dot{a}_{\bar{a}}=\left(\Sigma+\frac{\Theta}{3}\right)\left(\mathcal{A}_{a}+a_{a}\right)-\varepsilon_{a b} \mathcal{H}^{b} \\
2 \dot{\Sigma}_{\bar{a}}-\widehat{\mathcal{A}}_{a}=\delta_{a} \mathcal{A}-\left(\Sigma+\frac{4 \Theta}{3}\right) \Sigma_{a}-3 \Sigma \alpha_{a}-2 \mathcal{E}_{a}, \\
\dot{\mathcal{E}}_{\bar{a}}+\frac{1}{2} \varepsilon_{a b} \widehat{\mathcal{H}}^{b}=\frac{3}{4}\left(\Sigma-\frac{4 \Theta}{3}\right) \mathcal{E}_{a}+\frac{(3 \mathcal{E}-2 \mu-2 p)}{4} \Sigma_{a}+\frac{3}{4} \varepsilon_{a b} \delta^{b} \mathcal{H}-\frac{3 \mathcal{E}}{2} \alpha_{a}+\frac{1}{2} \varepsilon_{b c} \delta^{b} \mathcal{H}_{a}^{c}, \\
\dot{\mathcal{H}}_{\bar{a}}-\frac{1}{2} \varepsilon_{a b} \widehat{\mathcal{E}}^{b}=\frac{3}{4}\left(\Sigma-\frac{4 \Theta}{3}\right) \mathcal{H}_{a}-\frac{3}{4} \varepsilon_{a b} X^{b}-\frac{3 \mathcal{E}}{2} \varepsilon_{a b} \mathcal{A}^{b}+\frac{3 \mathcal{E}}{4} \varepsilon_{a b} a^{b}-\frac{1}{2} \varepsilon_{b c} \delta^{b} \mathcal{E}_{a \prime}^{c}, \\
\dot{\mathcal{E}}_{\{a b\}}-\varepsilon_{c\{a} \widehat{\mathcal{H}}_{b\}}^{c}=-\frac{3}{2}\left(\Sigma+\frac{2 \Theta}{3}\right) \mathcal{E}_{a b}-\varepsilon_{c\{a} \delta^{c} \mathcal{H}_{b\}}-\frac{(3 \mathcal{E}+\mu+p)}{2} \Sigma_{a b}, \\
\dot{\mathcal{H}}_{\{a b\}}+\varepsilon_{c\{a} \widehat{\mathcal{E}}_{b\}}{ }^{c}=-\frac{3}{2}\left(\Sigma+\frac{2 \Theta}{3}\right) \mathcal{H}_{a b}+\frac{3 \mathcal{E}}{2} \mathcal{E}_{c\{a} \zeta_{b\}}^{c}+\varepsilon_{c\{a} \delta^{c} \mathcal{E}_{b\}} .
\end{gathered}
$$

The pure propagation equations are

$$
\begin{gathered}
\widehat{\phi}=-\left(\Sigma-\frac{2 \Theta}{3}\right)\left(\Sigma+\frac{\Theta}{3}\right)+\delta^{a} a_{a}-\mathcal{E}-\frac{2(\mu+\Lambda)}{3}, \\
2 \widehat{\xi}=\varepsilon^{a b} \delta_{a} a_{b},
\end{gathered}
$$




$$
\begin{gathered}
\widehat{\mathcal{H}}=-\delta^{a} \mathcal{H}_{a}, \\
\widehat{\mathcal{A}}_{a}=\delta_{a} \mathcal{A}, \\
\widehat{p}_{\bar{a}}=-(\mu+p) \delta_{a} \mathcal{A}, \\
\frac{\widehat{\mu}_{\bar{a}}}{3}-\widehat{X}_{\bar{a}}=\frac{3 \mathcal{E}}{2} \delta_{a} \phi+\delta_{a} \delta^{b} \mathcal{E}_{b}, \\
\frac{2}{3} \widehat{W}_{\bar{a}}-\widehat{V}_{\bar{a}}=\frac{3 \Sigma}{2} \delta_{a} \phi+\delta_{a} \delta^{b} \Sigma_{b}, \\
\widehat{\Sigma}_{\bar{a}}=\frac{1}{2}\left(V_{a}+\frac{4}{3} W_{a}\right)-\frac{3 \Sigma}{2} a_{a}-\delta^{b} \Sigma_{a b}, \\
2 \widehat{\mathcal{E}}_{\bar{a}}=X_{a}-3 \mathcal{E} a_{a}-3 \Sigma \varepsilon_{a b} \mathcal{H}^{b}-2 \delta^{b} \mathcal{E}_{a b}+\frac{2}{3} \mu_{a}, \\
2 \widehat{\mathcal{H}}_{\bar{a}}=\delta_{a} \mathcal{H}-3 \mathcal{E} \varepsilon_{a b} \Sigma^{b}+3 \Sigma \varepsilon_{a b} \mathcal{E}^{b}-2 \delta^{b} \mathcal{H}_{a b}, \\
\widehat{\Sigma}_{\{a b\}}=\delta_{\{a} \Sigma_{b\}}+\frac{3 \Sigma}{2} \zeta_{a b}-\varepsilon_{c\{a} \mathcal{H}_{b\}}{ }^{c}, \\
\widehat{\zeta}_{\{a b\}}=\left(\Sigma+\frac{\Theta}{3}\right) \Sigma_{a b}+\delta_{\{a} a_{b\}}-\mathcal{E}_{a b} .
\end{gathered}
$$

Finally, the constraints are

$$
\begin{gathered}
\varepsilon^{a b} \delta_{a} \mathcal{A}_{b}=0, \\
p_{a}=-(\mu+p) \mathcal{A}_{a}, \\
\varepsilon^{a b} \delta_{a} \Sigma_{b}=-3 \Sigma \xi+\mathcal{H}, \\
\varepsilon_{a b} \delta^{b} \xi+\delta^{b} \zeta_{a b}-\frac{\delta_{a} \phi}{2}=\frac{1}{2}\left(\Sigma-\frac{2 \Theta}{3}\right) \Sigma_{a}+\mathcal{E}_{a}, \\
\left(V_{a}-\frac{2}{3} W_{a}\right)+2 \delta^{b} \Sigma_{a b}=-2 \varepsilon_{a b} \mathcal{H}^{b} .
\end{gathered}
$$

\section{Appendix C. Evolution and Propagation Equations and Constraints for $\phi \neq 0$}

Here, the evolution and propagation equations and constraints are given for the exceptional case where $\phi \neq 0$ on the background, corresponding to the negatively curved Friedmann universe. The set $S^{(0)}=\{\Theta, \mu, p, \phi\}$ gives the quantities which are nonzero on the background. We define corresponding first-order quantities

$$
\mu_{a}=\delta_{a} \mu, p_{a}=\delta_{a} p, W_{a}=\delta_{a} \Theta, \phi_{a}=\delta_{a} \phi,
$$

which vanish on the background. As before, we let $V_{a}=\delta_{a} \Sigma$ and $X_{a}=\delta_{a} \mathcal{E}$. Vorticity-free perturbations hence are given by the following nonzero first-order quantities (which all vanish on the background):

$$
S^{(1)} \equiv\left\{\phi_{a}, W_{a}, \mu_{a}, p_{a}, \mathcal{A}, \mathcal{A}_{a}, \Sigma, \Sigma_{a}, \Sigma_{a b}, \mathcal{E}, \mathcal{E}_{a}, \mathcal{E}_{a b}, \mathcal{H}, \mathcal{H}_{a}, \mathcal{H}_{a b}, a_{b}, \alpha_{a}, \xi, \zeta_{a b}\right\}
$$

The following evolution equations then hold on the perturbed spacetimes:

$$
\begin{aligned}
\dot{\phi}_{\bar{a}}=\dot{\phi} \mathcal{A}_{a}-\frac{2 \Theta}{3} \phi_{a} & +\left(V_{a}-\frac{2}{3} W_{a}\right) \frac{\phi}{2}+\frac{2 \Theta}{3} \delta_{a} \mathcal{A}+\delta_{a} \delta^{b} \alpha_{b}, \\
2 \dot{\xi} & =-\frac{2 \Theta}{3} \xi+\varepsilon^{a b} \delta_{a} \alpha_{b}+\mathcal{H}, \\
\dot{\mathcal{H}} & =-\Theta \mathcal{H}-\varepsilon^{a b} \delta_{a} \mathcal{E}_{b},
\end{aligned}
$$




$$
\begin{gathered}
\dot{\mu}_{\bar{a}}=-\frac{\Theta}{3} \mu_{a}-\Theta\left(\mu_{a}+p_{a}\right)-(\mu+p) W_{a}+\dot{\mu} \mathcal{A}_{a}, \\
\dot{\mathcal{E}}=-\Theta \mathcal{E}-\frac{1}{2}(\mu+p) \Sigma+\varepsilon^{a b} \delta_{a} \mathcal{H}_{b}, \\
\dot{\Sigma}_{\{a b\}}=-\frac{2 \Theta}{3} \Sigma_{a b}+\delta_{\{a} \mathcal{A}_{b\}}-\mathcal{E}_{a b}, \\
\dot{\zeta}_{\{a b\}}=-\frac{\Theta}{3} \zeta_{a b}+\delta_{\{a} \alpha_{b\}}-\varepsilon_{c\{a} \mathcal{H}_{b\}}{ }^{c} .
\end{gathered}
$$

The equations containing both propagation and evolution contributions are

$$
\begin{gathered}
\dot{\Sigma}-\frac{2}{3} \widehat{\mathcal{A}}=-\frac{1}{3} \phi \mathcal{A}-\frac{2}{3} \Theta \Sigma-\frac{1}{3} \delta_{a} \mathcal{A}^{a}-\mathcal{E}, \\
\dot{W}_{\bar{a}}-\delta_{a} \widehat{\mathcal{A}}=-\Theta W_{a}-\frac{1}{2}\left(\mu_{a}+3 p_{a}\right)+\dot{\Theta} \mathcal{A}_{a}+\delta_{a} \delta^{b} \mathcal{A}_{b}+\phi \delta_{a} \mathcal{A}, \\
\widehat{\alpha}_{\bar{a}}-\dot{a}_{\bar{a}}=\frac{\Theta}{3}\left(\mathcal{A}_{a}+a_{a}\right)-\varepsilon_{a b} \mathcal{H}-\frac{1}{2} \phi \alpha_{a}, \\
2 \dot{\Sigma}_{\bar{a}}-\widehat{\mathcal{A}}_{a}=\delta_{a} \mathcal{A}-\frac{4 \Theta}{3} \Sigma_{a}-2 \mathcal{E}_{a}-\frac{1}{2} \phi \mathcal{A}_{a}, \\
\dot{\mathcal{E}}_{\bar{a}}+\frac{1}{2} \varepsilon_{a b} \widehat{\mathcal{H}}^{b}=-\Theta \mathcal{E}_{a}-\frac{1}{2}(\mu+p) \Sigma_{a}+\frac{3}{4} \varepsilon_{a b} \delta^{b} \mathcal{H}+\frac{1}{2} \varepsilon_{b c} \delta^{b} \mathcal{H}_{a}^{c}-\frac{1}{4} \phi \varepsilon_{a b} \mathcal{H}^{b}, \\
\dot{\mathcal{H}}_{\bar{a}}-\frac{1}{2} \varepsilon_{a b} \widehat{\mathcal{E}}^{b}=-\Theta \mathcal{H}_{a}-\frac{3}{4} \mathcal{E}_{a b} X^{b}-\frac{1}{2} \varepsilon_{b c} \delta^{b} \mathcal{E}_{a}^{c}+\frac{1}{4} \phi \varepsilon_{a b} \mathcal{E}^{b}, \\
\dot{\mathcal{E}}_{\{a b\}}-\varepsilon_{c\{a} \widehat{\mathcal{H}}_{b\}}{ }^{c}=-\Theta \mathcal{E}_{a b}-\varepsilon_{c\{a} \delta^{c} \mathcal{H}_{b\}}-\frac{(\mu+p)}{2} \Sigma_{a b}+\frac{1}{2} \phi \varepsilon_{c\{a} \mathcal{H}_{b\}}^{c}, \\
\dot{\mathcal{H}}_{\{a b\}}+\varepsilon_{c\{a} \widehat{\mathcal{E}}_{b\}}^{c}=-\Theta \mathcal{H}_{a b}+\varepsilon_{c\{a} \delta^{c} \mathcal{E}_{b\}}-\frac{1}{2} \phi \varepsilon_{c\{a} \mathcal{E}_{b\}}^{c} .
\end{gathered}
$$

The pure propagation equations are

$$
\begin{gathered}
\widehat{\phi}_{\bar{a}}=\frac{1}{3}\left(V_{a}+\frac{4}{3} W_{a}\right) \Theta+\delta_{a} \delta^{b} a_{b}-X_{a}-\frac{2}{3} \mu_{a}-\frac{3}{2} \phi \phi_{a}, \\
2 \widehat{\xi}=\varepsilon^{a b} \delta_{a} a_{b}-2 \phi \xi, \\
\widehat{\mathcal{H}}=-\delta^{a} \mathcal{H}_{a}-\frac{3}{2} \phi \mathcal{H}, \\
\widehat{\mathcal{A}}_{a}=\delta_{a} \mathcal{A}-\frac{1}{2} \phi \mathcal{A}_{a}, \\
\widehat{p}_{\bar{a}}=-(\mu+p) \delta_{a} \mathcal{A}-\frac{1}{2} \phi p, \\
\frac{\widehat{\mu}_{\bar{a}}}{3}-\widehat{X}_{\bar{a}}=\delta_{a} \delta^{b} \mathcal{E}_{b}+2 \phi X_{a}-\frac{1}{6} \phi \mu_{a \prime} \\
\frac{2}{3} \widehat{W}_{\bar{a}}-\widehat{V}_{\bar{a}}=\frac{3 \Sigma}{2} \delta_{a} \phi+\delta_{a} \delta^{b} \Sigma_{b}+2 \phi V_{a}-\frac{1}{3} \phi W_{a}, \\
\widehat{\Sigma}_{\bar{a}}=\frac{1}{2}\left(V_{a}+\frac{4}{3} W_{a}\right)-\delta^{b} \Sigma_{a b}-\frac{3}{2} \phi \Sigma_{a b}, \\
2 \widehat{\mathcal{E}}_{\bar{a}}=X_{a}-2 \delta^{b} \mathcal{E}_{a b}+\frac{2}{3} \mu_{a}-3 \phi \mathcal{E}_{a}, \\
2 \widehat{\mathcal{H}}_{\bar{a}}=\delta_{a} \mathcal{H}-2 \delta^{b} \mathcal{H}_{a b}-3 \phi \mathcal{H}_{a},
\end{gathered}
$$




$$
\begin{aligned}
& \widehat{\Sigma}_{\{a b\}}=\delta_{\{a} \Sigma_{b\}}-\varepsilon_{c\{a} \mathcal{H}_{b\}}{ }^{c}-\frac{1}{2} \phi \Sigma_{a b}, \\
& \widehat{\zeta}_{\{a b\}}=\frac{\Theta}{3} \Sigma_{a b}+\delta_{\{a} a_{b\}}-\mathcal{E}_{a b}-\phi \zeta_{a b} .
\end{aligned}
$$

Finally, the constraints are

$$
\begin{gathered}
\varepsilon^{a b} \delta_{a} \mathcal{A}_{b}=0 \\
p_{a}=-(\mu+p) \mathcal{A}_{a}, \\
\varepsilon^{a b} \delta_{a} \Sigma_{b}=\mathcal{H} \\
\varepsilon_{a b} \delta^{b} \xi+\delta^{b} \zeta_{a b}-\frac{\phi_{a}}{2}=-\frac{\Theta}{3} \Sigma_{a}+\mathcal{E}_{a}, \\
\left(V_{a}-\frac{2}{3} W_{a}\right)+2 \delta^{b} \Sigma_{a b}=-2 \varepsilon_{a b} \mathcal{H}^{b}-\phi \Sigma_{a} .
\end{gathered}
$$

\section{Appendix D. Harmonics}

In this Appendix we enlist a set of identities for the even $Q_{a}^{k_{\perp}}$ and odd $\bar{Q}_{a}^{k_{\perp}}$ vector harmonics, including the orthogonality relations

$$
N^{a b} Q_{a}^{k_{\perp}} \bar{Q}_{b}^{k_{\perp}}=0
$$

the algebraic relations

$$
Q_{a}^{k_{\perp}}=-\varepsilon_{a}{ }^{b} \bar{Q}_{b}^{k_{\perp}}, \quad \bar{Q}_{a}^{k_{\perp}}=\varepsilon_{a}{ }^{b} Q_{b}^{k_{\perp}},
$$

and the differential relations

$$
\begin{gathered}
\dot{Q}_{a}^{k_{\perp}}=\widehat{Q}_{a}^{k_{\perp}}=0, \quad \dot{\bar{Q}}_{a}^{k_{\perp}}=\widehat{\bar{Q}}_{a}^{k_{\perp}}=0, \\
\delta^{2} Q_{a}^{k_{\perp}}=\frac{\mathcal{R} a_{2}^{2}-2 k_{\perp}^{2}}{2 a_{2}^{2}} Q_{a}^{k_{\perp}}, \quad \delta^{2} \bar{Q}_{a}^{k_{\perp}}=\frac{\mathcal{R} a_{2}^{2}-2 k_{\perp}^{2}}{2 a_{2}^{2}} \bar{Q}_{a}^{k_{\perp}}, \\
\delta^{a} Q_{a}^{k_{\perp}}=-\frac{k_{\perp}^{2}}{a_{2}} Q^{k_{\perp}}, \quad \delta^{a} \bar{Q}_{a}^{k_{\perp}}=0, \\
\varepsilon^{a b} \delta_{a} Q_{b}^{k_{\perp}}=0, \quad \varepsilon^{a b} \delta_{a} \bar{Q}_{b}^{k_{\perp}}=\frac{k_{\perp}^{2}}{a_{2}} Q^{k_{\perp},}
\end{gathered}
$$

where the 2-curvature $\mathcal{R}$ is given by Equation (18).

The even and odd tensor spherical harmonics obey the orthogonality relations

$$
N^{a b} N^{c d} Q_{a c}^{k_{\perp}} \bar{Q}_{b d}^{k_{\perp}}=0,
$$

the algebraic relations

$$
Q_{a b}^{k_{\perp}}=\varepsilon_{\{a}{ }^{c} \bar{Q}_{b\} c}^{k_{\perp}}, \quad \bar{Q}_{a b}^{k_{\perp}}=-\varepsilon_{\{a}{ }^{c} Q_{b\} c}^{k_{\perp}},
$$

and the differential relations

$$
\begin{gathered}
\dot{Q}_{a b}^{k_{\perp}}=\widehat{Q}_{a b}^{k_{\perp}}=0, \quad \dot{\bar{Q}}_{a b}^{k_{\perp}}=\hat{\bar{Q}}_{a b}^{k_{\perp}}=0, \\
\delta^{2} Q_{a b}^{k_{\perp}}=\frac{2 \mathcal{R} a_{2}^{2}-k_{\perp}^{2}}{a_{2}^{2}} Q_{a b}^{k_{\perp}}, \quad \delta^{2} \bar{Q}_{a b}^{k_{\perp}}=\frac{2 \mathcal{R} a_{2}^{2}-k_{\perp}^{2}}{a_{2}^{2}} \bar{Q}_{a b}^{k_{\perp},} \\
\delta^{b} Q_{a b}^{k_{\perp}}=\frac{\mathcal{R} a_{2}^{2}-k_{\perp}^{2}}{a_{2}^{2}} Q_{a}^{k_{\perp}}, \quad \delta^{b} \bar{Q}_{a b}^{k_{\perp}}=-\frac{\mathcal{R} a_{2}^{2}-k_{\perp}^{2}}{a_{2}^{2}} \bar{Q}_{a}^{k_{\perp}},
\end{gathered}
$$




$$
\begin{aligned}
& \varepsilon_{a}^{c} \delta^{b} Q_{b c}^{k_{\perp}}=\frac{\mathcal{R} a_{2}^{2}-k_{\perp}^{2}}{a_{2}^{2}} \bar{Q}_{a}^{k_{\perp}}, \quad \varepsilon_{a}^{c} \delta^{b} \bar{Q}_{b c}^{k_{\perp}}=\frac{\mathcal{R} a_{2}^{2}-k_{\perp}^{2}}{a_{2}^{2}} Q_{a}^{k_{\perp}}, \\
& \varepsilon^{b c} \delta_{b} Q_{a c}^{k_{\perp}}=\frac{\mathcal{R} a_{2}^{2}-k_{\perp}^{2}}{a_{2}^{2}} \bar{Q}_{a}^{k_{\perp}}, \quad \varepsilon^{b c} \delta_{b} \bar{Q}_{a c}^{k_{\perp}}=\frac{\mathcal{R} a_{2}^{2}-k_{\perp}^{2}}{a_{2}^{2}} Q_{a}^{k_{\perp}} .
\end{aligned}
$$

\section{Appendix E. Harmonic Coefficients}

The perturbation variables coupled to $\mu_{k_{\|} k_{\perp}}^{V}, \Sigma_{k_{\|} k_{\perp}}^{T}, \mathcal{E}_{k_{\|}}^{T} k_{\perp}$, and $\overline{\mathcal{H}}_{k_{\|} k_{\perp}}^{T}$ are

$$
\begin{aligned}
& \mathcal{A}_{k_{\|} k_{\perp}}^{V}=\frac{a_{1}}{i k_{\|}} \frac{\mathcal{A}_{k_{\|} k_{\perp}}^{S}}{a_{2}}=-\frac{c_{s}^{2}}{\mu+p} \mu_{k_{\|} k_{\perp}}^{V} \\
& \frac{i k_{\|}}{a_{1}} \zeta_{k_{\|} k_{\perp}}=\left(\Sigma+\frac{\Theta}{3}\right) \Sigma_{k_{\|} k_{\perp}}^{T}-\mathcal{E}_{k_{\|} k_{\perp}}^{T} \\
& \frac{i k_{\|}}{a_{1} a_{2}} \Sigma_{k_{\|} k_{\perp}}^{V}=-\left(B+\frac{\mathcal{R} a_{2}^{2}-k_{\perp}^{2}}{a_{2}^{2}}\right) \frac{\Sigma_{k_{\|} k_{\perp}}^{T}}{2}+\frac{3}{2} \Sigma \mathcal{E}_{k_{\|} k_{\perp}}^{T}-i \frac{k_{\|}}{a_{1}} \overline{\mathcal{H}}_{k_{\|} k_{\perp}}^{T}, \\
& \frac{2 \overline{\mathcal{H}}_{k_{\|} k_{\perp}}^{V}}{3 a_{2}}=-\frac{\Sigma}{a_{2} B} \mu_{k_{\|} k_{\perp}}^{V}+\Sigma C \mathcal{E}_{k_{\|} k_{\perp}}^{T}-\mathcal{E} \Sigma_{k_{\|} k_{\perp}}^{T}-\frac{i k_{\|}}{a_{1}} J \frac{\overline{\mathcal{H}}_{k_{\|} k_{\perp}}^{T}}{3}, \\
& \frac{V_{k_{\|} k_{\perp}}^{V}}{a_{2}}=-\frac{2 \Sigma}{a_{2} B} \mu_{k_{\|} k_{\perp}}^{V}-\frac{B}{3}(1+2 C) \Sigma_{k_{\|} k_{\perp}}^{T}+\Sigma(1+2 C) \mathcal{E}_{k_{\|} k_{\perp}}^{T}-\frac{2}{3} \frac{i k_{\|}}{a_{1}}(1+J) \overline{\mathcal{H}}_{k_{\|} k_{\perp}}^{T}, \\
& \frac{W_{k_{\|} k_{\perp}}^{V}}{a_{2}}=\frac{3 \Sigma}{2 a_{2} B} \mu_{k_{\|} k_{\perp}}^{V}+\left(3 \mathcal{E}+\frac{\mathcal{R} a_{2}^{2}-k_{\perp}^{2}}{a_{2}^{2}}-B\right) \frac{\Sigma_{k_{\|} k_{\perp}}^{T}}{2} \\
& +\frac{3 \Sigma}{2}(1-C) \mathcal{E}_{k_{\|} k_{\perp}}^{T}-\frac{i k_{\|}}{2 a_{1}}(2-J) \overline{\mathcal{H}}_{k_{\|} k_{\perp}}^{T}, \\
& \frac{i k_{\|}}{a_{1}} \frac{\phi_{k_{\|} k_{\perp}}^{S}}{a_{2}^{2}}=-\frac{k_{\|}^{2}}{a_{1}^{2}} \frac{2}{a_{2} B} \mu_{k_{\|} k_{\perp}}^{V}-\frac{B L}{3} \Sigma_{k_{\|} k_{\perp}}^{T}+\left(L \Sigma-\frac{\mathcal{R} a_{2}^{2}-k_{\perp}^{2}}{a_{2}^{2} B} \frac{k_{\perp}^{2}}{a_{2}^{2}}\right) \mathcal{E}_{k_{\|} k_{\perp}}^{T}-\frac{i k_{\|}}{a_{1}} \frac{2 L}{3} \overline{\mathcal{H}}_{k_{\|} k_{\perp}}^{T}, \\
& \frac{i k_{\|}}{a_{1} a_{2}} \alpha_{k_{\|} k_{\perp}}^{V}=-\left[\frac{3 \Sigma}{2 B}+\left(\Sigma+\frac{\Theta}{3}\right) \frac{c_{s}^{2}}{\mu+p}\right] \frac{\mu_{k_{\|} k_{\perp}}^{V}}{a_{2}} \\
& -\frac{3 \mathcal{E}}{2} \Sigma_{k_{\|} k_{\perp}}^{T}+\frac{3 \Sigma}{2} C \mathcal{E}_{k_{\|} k_{\perp}}^{T}-\frac{i k_{\|}}{2 a_{1}} J \overline{\mathcal{H}}_{k_{\|} k_{\perp}}^{T} \\
& \frac{X_{k_{\|} k_{\perp}}^{V}}{a_{2}}=\left[1-\frac{3}{B}\left(\frac{k_{\perp}^{2}}{a_{2}^{2}}+3 \mathcal{E}\right)\right] \frac{\mu_{\|_{\|} k_{\perp}}^{V}}{3 a_{2}}-\mathcal{E}\left(\Theta-\frac{3}{2} \Sigma\right) \Sigma_{k_{\|} k_{\perp}}^{T}+C\left(\frac{k_{\perp}^{2}}{a_{2}^{2}}+3 \mathcal{E}\right) \mathcal{E}_{k_{\|} k_{\perp}}^{T} \\
& +\frac{a_{1}}{i k_{\|}}\left[\mathcal{E}\left(\frac{1}{3} \Theta-\frac{1}{2} \Sigma\right) \frac{k_{\|}^{2}}{a_{1}^{2}}-\Sigma \frac{\mathcal{R} a_{2}^{2}-k_{\perp}^{2}}{4 a_{2}^{2}} \frac{k_{\perp}^{2}}{a_{2}^{2}}\right] \frac{6 \overline{\mathcal{H}}_{k_{\|}}^{T} k_{\perp}}{B}, \\
& \frac{i k_{\|}}{a_{1} a_{2}} \mathcal{E}_{k_{\|} k_{\perp}}^{V}=\frac{k_{\|}^{2}}{a_{1}^{2}} \frac{\mu_{\|\|^{2}}^{V} k_{\perp}}{a_{2} B}-\frac{3 \mathcal{E}}{2}\left(\Sigma+\frac{\Theta}{3}\right) \Sigma_{k_{\|} k_{\perp}}^{T}+\left(\frac{3 \mathcal{E}}{2}-C \frac{k_{\|}^{2}}{a_{1}^{2}}\right) \mathcal{E}_{k_{\|} k_{\perp}}^{T} \\
& -\frac{i k_{\|}}{a_{1}} \frac{3 \overline{\mathcal{H}}_{k_{\|}}^{T} k_{\perp}}{2 B}\left[2 \mathcal{E}\left(\Sigma+\frac{\Theta}{3}\right)+\Sigma \frac{\mathcal{R} a_{2}^{2}-k_{\perp}^{2}}{a_{2}^{2}}\right] \text {, }
\end{aligned}
$$

and which are coupled to $\overline{\mathcal{E}}_{k_{\|} k_{\perp}}^{T}, \mathcal{H}_{k_{\|} k_{\perp}}^{T}$ are

$$
\begin{aligned}
\mathcal{H}_{k_{\|} k_{\perp}}^{V} & =\frac{i k_{\|}}{a_{1}} \frac{a_{2}}{k_{\perp}^{2}} \mathcal{H}_{k_{\|} k_{\perp}}^{S}=-\frac{i k_{\|}}{a_{1}} \bar{\alpha}_{k_{\|} k_{\perp}}^{V}=\frac{i k_{\|}}{a_{1}} \bar{\Sigma}_{k_{\|} k_{\perp}}^{V} \\
& =\frac{\mathcal{R} a_{2}^{2}-k_{\perp}^{2}}{2 a_{2}} \bar{\Sigma}_{k_{\|} k_{\perp}}^{T}=\frac{\mathcal{R} a_{2}^{2}-k_{\perp}^{2}}{a_{2} B}\left(\frac{3 \Sigma}{2} \overline{\mathcal{E}}_{k_{\|} k_{\perp}}^{T}+\frac{i k_{\|}}{a_{1}} \mathcal{H}_{k_{\|} k_{\perp}}^{T}\right),
\end{aligned}
$$




$$
\begin{aligned}
\frac{B}{2} \bar{\zeta}_{k_{\|} k_{\perp}}^{T} & =\left(\Sigma+\frac{\Theta}{3}\right) \mathcal{H}_{k_{\|} k_{\perp}}^{T}-\frac{a_{1}}{i k_{\|}}\left(\frac{k_{\|}^{2}}{a_{1}^{2}}-\frac{\mathcal{R} a_{2}^{2}-k_{\perp}^{2}}{2 a_{2}^{2}}\right) \overline{\mathcal{E}}_{k_{\|} k_{\perp}}^{T}, \\
\overline{\mathcal{E}}_{k_{\|} k_{\perp}}^{V} & =\frac{\mathcal{R} a_{2}^{2}-k_{\perp}^{2}}{2 a_{2}}\left[\frac{a_{1}}{i k_{\|}}\left(1-\frac{9 \Sigma^{2}}{2 B}\right) \overline{\mathcal{E}}_{k_{\|} k_{\perp}}^{T}-\frac{3 \Sigma}{B} \mathcal{H}_{k_{\|} k_{\perp}}^{T}\right],
\end{aligned}
$$

where

$$
\begin{gathered}
B \equiv \frac{2 k_{\|}^{2}}{a_{1}^{2}}+\frac{k_{\perp}^{2}}{a_{2}^{2}}+\frac{9 \Sigma^{2}}{2}+3 \mathcal{E}=\frac{2 k_{\|}^{2}}{a_{1}^{2}}-\frac{\mathcal{R} a_{2}^{2}-k_{\perp}^{2}}{a_{2}^{2}}+3 \Sigma\left(\Sigma+\frac{\Theta}{3}\right), \\
C B \equiv\left(\frac{\mathcal{R} a_{2}^{2}-k_{\perp}^{2}}{a_{2}^{2}}+3 \mathcal{E}\right)=\Sigma\left(\Theta-\frac{3}{2} \Sigma\right)-\frac{k_{\perp}^{2}}{a_{2}^{2}} \\
L B \equiv \frac{1}{\Sigma}\left(2 C B \frac{k_{\|}^{2}}{a_{1}^{2}}+\frac{k_{\perp}^{2}}{a_{2}^{2}}\left(B+\frac{\mathcal{R} a_{2}^{2}-k_{\perp}^{2}}{a_{2}^{2}}\right)\right)=3 \Sigma\left(\frac{k_{\perp}^{2}}{a_{2}^{2}}-\frac{k_{\|}^{2}}{a_{1}^{2}}\right)+\Theta\left(\frac{2 k_{\|}^{2}}{a_{1}^{2}}+\frac{k_{\perp}^{2}}{a_{2}^{2}}\right), \\
J B \equiv \frac{\left(\mathcal{R} a_{2}^{2}-k_{\perp}^{2}\right) k_{\perp}^{2} a_{1}^{2}}{k_{\|}^{2} a_{2}^{4}}+2 C B=\left(\mathcal{R}-\frac{k_{\perp}^{2}}{a_{2}^{2}}\right)\left(\frac{2 k_{\|}^{2}}{a_{1}^{2}}+\frac{k_{\perp}^{2}}{a_{2}^{2}}\right) \frac{a_{1}^{2}}{k_{\|}^{2}}+6 \mathcal{E} \\
=\frac{k_{\perp}^{2} a_{1}^{2}}{k_{\|}^{2} a_{2}^{2}}\left(\mathcal{R}-\frac{2 k_{\|}^{2}}{a_{1}^{2}}-\frac{k_{\perp}^{2}}{a_{2}^{2}}\right)+2 \Sigma\left(\Theta-\frac{3}{2} \Sigma\right) .
\end{gathered}
$$

\section{Appendix F. Shear and Weyl Tensor Tensorial Waves in Friedmann Spacetime}

In Friedmann spacetimes the background equations [see Equations (3.29), (3.34) and (3.40) of [43], respectively, specified for Friedmann spacetimes] are:

$$
\begin{gathered}
\dot{\mu}+\Theta(\mu+p)=0, \\
\dot{\Theta}+\frac{\Theta^{2}}{3}+\frac{\mu+3 p}{2}-\Lambda=0, \\
\frac{\Theta^{2}}{3}+\frac{3 K}{a^{2}}=\mu+\Lambda,
\end{gathered}
$$

with curvature $6 K / a^{2}={ }^{(3)} R$ of 3-spaces of homogeneity, where ${ }^{(3)} R$ is the three-dimensional Ricci scalar.

In Friedmann spacetimes the perturbations are classified into scalar, vector, and tensor types. The pure tensor perturbations are characterized by vanishing of vorticity and of all gauge-invariant vectors and scalars at the first order $[53,55]$. Thus, the acceleration and the gradient of all scalars also vanish. Moreover we assume the perturbed energy momentum tensor to describe a barotropic perfect fluid.

The evolution and constraint equations for the shear and for the electric and magnetic parts of Weyl tensor in $1+3$ covariant formalism [see Equations (7)-(9), (12), (13) and (21), of [53], respectively] are:

$$
\begin{gathered}
\dot{\sigma}_{<a b>}+\frac{2}{3} \Theta \sigma_{a b}+E_{a b}=0, \\
\dot{E}_{<a b>}+\Theta E_{a b}-\operatorname{curl} H_{a b}+\frac{\mu+p}{2} \sigma_{a b}=0, \\
\dot{H}_{<a b>}+\Theta H_{a b}+\operatorname{curl} E_{a b}=0, \\
H_{a b}=\operatorname{curl} \sigma_{a b}, \\
D^{a} H_{a b}=0, \\
D^{a} E_{a b}=0,
\end{gathered}
$$


where the curl of a tensor $T_{a b}$ is given by

$$
\operatorname{curl}^{a b}=\varepsilon^{c d<a} D_{c} \sigma_{d}^{b>} .
$$

We expand all tensor quantities in terms of tensorial three-dimensional harmonics with even $Q_{a b}^{(k)}$ and odd $\bar{Q}_{a b}^{(k)}$ parities. These harmonics are related by a curl operation [see Equations (A19) and (A20) of [68], respectively, or Subsection 11.2.3 of [69]]

$$
\begin{aligned}
& \operatorname{curl} Q_{a b}^{(k)}=\frac{k}{a} \sqrt{1+\frac{3 K}{k^{2}}} \bar{Q}_{a b}^{(k)}, \\
& \operatorname{curl} \bar{Q}_{a b}^{(k)}=\frac{k}{a} \sqrt{1+\frac{3 K}{k^{2}}} Q_{a b}^{(k)} .
\end{aligned}
$$

The tensor harmonics are symmetric, trace-free and obey

$$
\begin{aligned}
& D^{2} Q_{a b}^{(k)}=-\frac{k^{2}}{a^{2}} Q_{a b}^{(k)}, \\
& D^{a} Q_{a b}^{(k)}=0, \dot{Q}_{a b}^{(k)}=0,
\end{aligned}
$$

and the same relations hold for $\bar{Q}_{a b}^{(k)}$ [see Appendix E and F of [70]]. Here, $k$ is discrete for closed Friedmann spacetimes while continous for flat and open universes [68]. From Equations (A121) and (A122) it follows that $Q_{a b}^{(k)}$ satisfies the relation

$$
\operatorname{curl} \operatorname{curl} Q_{a b}^{(k)}=\frac{k^{2}}{a^{2}}\left(1+\frac{3 K}{k^{2}}\right) Q_{a b}^{(k)}
$$

and the same is true for $\bar{Q}_{a b}^{(k)}$.

All symmetric, trace-free and divergenceless first- order tensors are expanded as

$$
T_{a b}=\sum_{k}\left(T_{k} Q_{a b}^{(k)}+\bar{T}_{k} \bar{Q}_{a b}^{(k)}\right) .
$$

Note that sometimes some power of $k / a$ are included in the harmonic expansion $[55,68]$, and the convention used in Equation (A125) corresponds to that of [53]. Since the three-dimensional volume element occurs in the definition of $H_{a b}, \bar{H}_{k}$ belongs to the even parity sector, and $H_{k}$ to the odd parity sector.

By using the harmonic expansion the constraints (A118) and (A119) are satisfied, while Equation (A117) becomes

$$
\begin{aligned}
H_{k} & =\frac{k}{a} \sqrt{1+\frac{3 K}{k^{2}}} \bar{\sigma}_{k}, \\
\bar{H}_{k} & =\frac{k}{a} \sqrt{1+\frac{3 K}{k^{2}}} \sigma_{k} .
\end{aligned}
$$

Applying these, the evolution equations Equations (A114)-(A116) reduce to

$$
\begin{gathered}
\dot{\sigma}_{k}+\frac{2}{3} \Theta \sigma_{k}+E_{k}=0, \\
\dot{E}_{k}+\Theta E_{k}+\left[\frac{\mu+p}{2}-\frac{k^{2}}{a^{2}}\left(1+\frac{3 K}{k^{2}}\right)\right] \sigma_{k}=0, \\
\dot{H}_{k}+\Theta H_{k}+\frac{k}{a} \sqrt{1+\frac{3 K}{k^{2}}} \bar{E}_{k}=0 .
\end{gathered}
$$


Equations for the coefficients belonging to the odd parity can be obtained by interchanging the overbared and unoverbared variables, respectively. From Equations (A128)-(A130), the following wave equations can be derived for the shear, and the electric and magnetic parts of Weyl tensor:

$$
\begin{gathered}
\ddot{\sigma}_{k}+\frac{5}{3} \Theta \dot{\sigma}_{k}+\left[\frac{k^{2}}{a^{2}}\left(1+\frac{K}{2 k^{2}}\right)+\frac{\Theta^{2}}{6}+\frac{3}{2}(\Lambda-p)\right] \sigma_{k}=0, \\
\ddot{E}_{k}+q_{1} \dot{E}_{k}+q_{0} E_{k}=0, \\
\ddot{H}_{k}+\frac{7 \Theta}{3} \dot{H}_{k}+\left[\frac{k^{2}}{a^{2}}+\frac{2 \Theta^{2}}{3}+2(\Lambda-p)\right] H_{k}=0,
\end{gathered}
$$

with coefficients

$$
\begin{gathered}
q_{0}=\frac{k^{2}}{a^{2}}+\frac{2 \Theta^{2}}{3}+2(\Lambda-p)-\frac{\left(1+3 c_{s}^{2}\right)(\mu+p)}{\frac{2 k^{2}}{a^{2}}\left(1+\frac{3 K}{k^{2}}\right)-(\mu+p)} \frac{\Theta^{2}}{3}, \\
q_{1}=\frac{7 \Theta}{3}-\frac{\left(1+3 c_{s}^{2}\right)(\mu+p)}{\frac{2 k^{2}}{a^{2}}\left(1+\frac{3 K}{k^{2}}\right)-(\mu+p)} \frac{\Theta}{3} .
\end{gathered}
$$

Similar equations are valid for the other parity variables. In the derivation, the time derivatives of $\mu$ and $\Theta$ were eliminated by using Equations (A111) and (A112), respectively, the time derivate of $p$ by

$$
\dot{p}=c_{s}^{2} \dot{\mu},
$$

and finally the energy density $\mu$ is partly elimated by using (A113). Equations (A131)-(A133) correspond to Equations (92), (93) and (86), respectively, for $K=0$ and for the vanishing energy density 2-gradient.

\section{References}

1. Abbott, B.P.; Abbott, R.; Abbott, T.D.; Abernathy, M.R.; Acernese, F.; Ackley, K.; Adams, C.; Adams, T.; Addesso, P.; Adhikari, R.X.; et al. Observation of Gravitational Waves from a Binary Black Hole Merger. Phys. Rev. Lett. 2016, 116, 061102.

2. Komatsu, E.; Kogut, A.; Nolta, M.R.; Bennett, C.L.; Halpern, M.; Hinshaw, G.; Jarosik, N.; Limon, M.; Meyer, S.S.; Page, L.; et al. First-Year Wilkinson Microwave Anisotropy Probe (WMAP) Observations: Tests of Gaussianity. Astrophys. J. Suppl. 2003, 148, 119-134.

3. Spergel, D.N.; Bean, R.; Doré, O.; Nolta, M.R.; Bennett, C.L.; Dunkley, J.; Hinshaw, G.; Jarosik, N.; Komatsu, E.; Page, L.; et al. Three-Year Wilkinson Microwave Anisotropy Probe (WMAP) Observations: Implications for Cosmology. Astrophys. J. 2007, 170, 377-408.

4. Hinshaw, G.; Larson, D.; Komatsu, E.; Spergel, D.N.; Bennett, C.L.; Dunkley, J.; Nolta, M.R.; Halpern, M.; Hill, R.S.; Odegard, N.; et al. Nine-year Wilkinson Microwave Anisotropy Probe (WMAP) Observations: Cosmological Parameter Results. Astrophys. J. Suppl. 2013, 208, 19.

5. Ade, P.A.R.; Aghanim, N.; Armitage-Caplan, C.; Arnaud, M.; Ashdown, M.; Atrio-Barandela, F.; Aumont, J.; Baccigalupi, C.; Banday, A.J.; Barreiro, R.B.; et al. Planck 2013 results. XVI. Cosmological parameters. Astron. Astrophys. 2014, 571, A16.

6. Ade, P.A.R.; Aghanim, N.; Arnaud, M.; Ashdown, M.; Aumont, J.; Baccigalupi, C.; Banday, A.J.; Barreiro, R.B.; Bartlett, J.G.; Bartolo, N.; et al. Planck 2015 results. XIII. Cosmological parameters. Astron. Astrophys. 2015, 594, A13.

7. Bennett, C.L.; Halpern, M.; Hinshaw, G.; Jarosik, N.; Kogut, A.; Limon, M.; Meyer, S.S.; Page, L.; Spergel, D.N.; Tucker, G.S.; et al. First-Year Wilkinson Microwave Anisotropy Probe (WMAP) Observations: Preliminary Maps and Basic Results. Astrophys. J. Suppl. 2003, 148, 1-27.

8. De Oliveira-Costa, A.; Tegmark, M.; Zaldarriaga, M.; Hamilton, A. Significance of the largest scale CMB fluctuations in WMAP. Phys. Rev. D 2004, 69, 063516. 
9. Vielva, P.; Martínez-González, E.; Barreiro, R.B.; Sanz, J.L.; Cayón, L. Detection of Non-Gaussianity in the Wilkinson Microwave Anisotropy Probe First-Year Data Using Spherical Wavelets. Astrophys. J. 2004, 609, $22-34$.

10. Ade, P.A.R.; Aghanim, N.; Akrami, Y.; Aluri, P.K.; Arnaud, M.; Ashdown, M.; Aumont, J.; Baccigalupi, C.; Banday, A.J.; Barreiro, R.B.; et al. Planck 2015 results. XVI. Isotropy and statistics of the CMB. Astron. Astrophys. 2016, 594, A16.

11. Padmanabhan, T. Accelerated expansion of the universe driven by tachyonic matter. Phys. Rev. D 2002, $66,021301$.

12. Frolov, A.V.; Kofman, L.; Starobinsky, A.A. Prospects and problems of tachyon matter cosmology. Phys. Lett. B 2002, 545, 8-16.

13. Csáki, C.; Kaloper; N.; Terning, J. The accelerated acceleration of the Universe. J. Cosmol. Astropart. Phys. 2006, 2006, 022.

14. Gorini, V.; Kamenshchik, A.Y.; Moschella, U.; Pasquier, V. Tachyons, scalar fields and cosmology. Phys. Rev. D 2004, 69, 123512.

15. Yoo, C.-M.; Nakao, K.-I.; Sasaki, M. CMB observations in LTB universes: Part I: Matching peak positions in the CMB spectrum. J. Cosmol. Astropart. Phys. 2010, 2010, 012.

16. Nojiri, S.; Odintsov, S.D. Unified cosmic history in modified gravity: From $F(R)$ theory to Lorentz non-invariant models. Phys. Rep. 2011, 505, 59-144.

17. Lombriser, L.; Slosar, A.; Seljak, U.; Hu, W. Constraints on $\mathrm{f}(\mathrm{R})$ gravity from probing the large-scale structure. Phys. Rev. D 2012, 85, 124038.

18. Tsujikawa, S. Quintessence: A Review. Classical Quantum Gravity 2013, 30, 214003.

19. Novosyadlyj, B.; Sergijenko, O.; Durrer, R.; Pelykh, V. Constraining the dynamical dark energy parameters: Planck-2013 vs. WMAP9. J. Cosmol. Astropart. Phys. 2014, 2014, 030.

20. Keresztes, Z.; Gergely, L. Á. Combined cosmological tests of a bivalent tachyonic dark energy scalar field model. J. Cosmol. Astropart. Phys. 2014, 2014, 026.

21. Gergely, L.Á.; Tsujikawa, S. Effective field theory of modified gravity with two scalar fields: Dark energy and dark matter. Phys. Rev. D 2014, 89, 064059.

22. Denkiewicz, T. Dark energy and dark matter perturbations in singular universes. J. Cosmol. Astropart. Phys. 2015, 2015, 037

23. McClure, M.L.; Dyer, C.C. Anisotropy in the Hubble constant as observed in the HST extragalactic distance scale key project results. New Astron. 2007, 12, 533-543.

24. Wiltshire, D.L.; Smale, P.R.; Mattsson, T.; Watkins, R. Hubble flow variance and the cosmic rest frame. Phys. Rev. D 2013, 88, 083529.

25. Cai, R.-G.; Tuo, Z.-L. Direction dependence of the deceleration parameter. J. Cosmol. Astropart. Phys. 2012, 2012,004 .

26. Doroshkevich, A.G.; Zel'dovich, Y.B.; Novikov, I.D. Perturbations in an anisotropic homogeneous universe. Zh. Ehksp. Teor. Fiz. 1971, 60, 3-8.

27. Perko, T.E.; Matzner, A.; Shepley, L.C. Galaxy Formation in Anisotropic Cosmologies. Phys. Rev. D 1972, 6, 969-983.

28. Tomita, K.; Den, M. Gauge-invariant perturbations in anisotropic homogeneous cosmological models. Phys. Rev. D 1986, 34, 3570-3583.

29. Gümrükçüoğlu, A.E.; Contaldi, C.R.; Peloso, M. Inflationary perturbations in anisotropic background and their imprint on the cosmic microwave background. J. Cosmol. Astropart. Phys. 2007, 2007, 005.

30. Periera, T.S.; Pitrou, C.; Uzan, J.-P. Theory of cosmological perturbations in an anisotropic universe. J. Cosmol. Astropart. Phys. 2007, 2007, 006.

31. Pitrou, C.; Periera, T.S.; Uzan, J.-P. Predictions from an anisotropic inflationary era. J. Cosmol. Astropart. Phys. 2008, 2008, 004.

32. Lifshitz, E.M.; Khalatnikov, I.M. Investigations in relativistic cosmology. Adv. Phys. 1963, 12, $185-249$.

33. Bardeen, J.M. Gauge-invariant cosmological perturbations. Phys. Rev. D 1980, 22, 1882-1905.

34. Stewart, J.M. Perturbations of Friedmann-Robertson-Walker cosmological models. Classical Quantum Gravity 1990, 7, 1169-1180.

35. Hawking, S.W. Perturbations of an Expanding Universe. Astrophys. J. 1966, 145, 544-554.

36. Olson, D.W. Density perturbations in cosmological models. Phys. Rev. D 1976, 14, 327-331.

37. Ellis, G.F.R.; Bruni, M. Covariant and gauge-invariant approach to cosmological density fluctuations. Phys. Rev. D 1989, 40, 1804-1818. 
38. Bruni, M.; Dunsby, P.K.S.; Ellis, G.F.R. Cosmological perturbations and the physical meaning of gauge-invariant variables. Astrophys. J. 1992, 395, 34-53.

39. Dunsby, P.K.S. Covariant perturbations of anisotropic cosmological models. Phys. Rev. D 1993, 48, 3562-3576.

40. Osano, B. Beyond the Standard Model of Cosmology of Cosmology: A Perturbative Approach. Ph.D. Thesis, University of Cape Town, Cape Town, South Africa, 2007.

41. Osano, B. The Decoupling of Scalar-Modes from a Linearly Perturbed Dust-Filled Bianchi Type-I Model. Chin. Phys. Lett. 2014, 31, 010402.

42. Ellis, G.F.R.; van Elst, H. Cosmological models. NATO Adv. Study Inst. Ser. C. Math. Phys. Sci. 1999, 541, 1-116.

43. Ellis, G.F.R. Cosmological Models. In Modern Cosmology; Moschella, U., Gorini, V., Bonometto, S., Eds.; Taylor \& Francis: Milton Park, UK, 2002; pp. 108-158.

44. Ellis, G.F.R.; Hwang, J.; Bruni, M. Covariant and gauge-independent perfect-fluid Robertson-Walker perturbations. Phys. Rev. D 1989, 40, 1819-1826.

45. Clarkson, C. A covariant approach for perturbations of rotationally symmetric spacetimes. Phys. Rev. D 2007, 76,104034 .

46. Betschart, G.; Clarkson, C. Scalar field and electromagnetic perturbations on Locally Rotationally Symmetric spacetimes. Classical Quantum Gravity 2004, 21, 5587-5607.

47. Clarkson, C.A.; Barrett, R.K. Covariant Perturbations of Schwarzschild Black Holes. Classical Quantum Gravity 2003, 20, 3855-3884.

48. Keresztes, Z.; Gergely, L.Á. Covariant gravitational dynamics in 3+1+1 dimensions. Classical Quantum Gravity 2010, 27, 105009.

49. Stewart, J.M.; Walker, M. Perturbations of space-times in general relativity. Proc. R. Soc. Lond. A 1974, 341, 49-74.

50. Keresztes, Z.; Forsberg, M.; Bradley, M.; Dunsby, P.K.S.; Gergely, L.Á. Gravitational, shear and matter waves in Kantowski-Sachs cosmologies. J. Cosmol. Astropart. Phys. 2015, 2015, 042.

51. Van Elst, H.; Ellis, G.F.R. The Covariant Approach to LRS Perfect Fluid Spacetime Geometries. Classical Quantum Gravity 1996, 13, 1099-1128.

52. Marklund, M.; Bradley, M. Invariant construction of solutions to Einstein's field equations—LRS perfect fluids II. Classical Quantum Gravity 1999, 16, 1577-1597.

53. Dunsby, P.K.S.; Bassett, B.A.C.; Elllis, G.F.R. Covariant analysis of gravitational waves in a cosmological context. Classical Quantum Gravity 1997, 14, 1215-1222.

54. Challinor, A. Microwave background anistropies from gravitational waves: The $1+3$ covariant approach. Classical Quantum Gravity 2000, 17, 871-889.

55. Challinor, A.; Lasenby, A. Cosmic Microwave Background Anisotropies in the Cold Dark Matter Model: A Covariant and Gauge-invariant Approach. Astrophys. J. 1999, 513, 1-22.

56. Gebbie, T.; Ellis, G.F.R. $1+3$ Covariant Cosmic Microwave Background anisotropies I: Algebraic Relations for Mode and Multipole Expansions. Ann. Phys. 2000, 282, 285-320.

57. Gebbie, T.; Dunsby, P.K.S.; Ellis, G.F.R. $1+3$ Covariant Cosmic Microwave Background anisotropies II: The almost-Friedmann Lemaitre model. Annals Phys. 2000, 282, 321-394

58. Marteens, R. Linearization instability of gravity waves? Phys. Rev. D 1997, 55, 463-467.

59. Tsagas, C.G.; Challinor, A.; Maartens, R. Relativistic cosmology and large-scale structure. Phys. Rep. 2008, 465, 61-147.

60. Marklund, M. Invariant construction of solutions to Einstein's field equations-LRS perfect fluids I. Classical Quantum Gravity 1997, 14, 1267-1284.

61. Bradley, M.; Marklund, M. Finding solutions to Einstein's equations in terms of invariant objects. Classical Quantum Gravity 1996, 13, 3021-3037.

62. Bradley, M.; Karlhede, A. On the curvature description of gravitational fields. Classical Quantum Gravity 1990, 7, 449-463.

63. Bradley, M.; Dunsby, P.K.S.; Forsberg, M.; Keresztes, Z. Density growth in Kantowski-Sachs cosmologies with cosmological constant. Classical Quantum Gravity 2012, 29, 095023.

64. Clarkson, C.A.; Marklund, M.; Betschart, G.; Dunsby, P.K.S. The electromagnetic signature of black hole ring-down. Astrophys. J. 2004, 613, 492-505. 
65. Burston, R.B. $1+1+2$ gravitational perturbations on LRS class II space-times: Decoupling GEM tensor harmonic amplitudes. Classical Quantum Gravity 2008, 25, 075004.

66. Thorne, K.P. Multipole expansions of gravitational radiation. Rev. Mod. Phys. 1980, 52, 299-339.

67. Harrison, E.R. Normal modes of vibrations of the universe. Rev. Mod. Phys. 1967, 39, 862-882.

68. Challinor, A. Microwave background polarization in cosmological models. Phys. Rev. D 2000, 62, 043004.

69. Ellis, G.F.R.; Maartens, R.; MacCallum, M.A.H. Relativistic Cosmology; Cambridge University Press: Cambridge, UK, 2012.

70. Ullrich, P. Exact and Perturbed Friedmann-Lemaître Cosmologies. Master's Thesis, University of Waterloo, Waterloo, ON, Canada, 2007.

71. Isaacson, R.A. Gravitational Radiation in the Limit of High Frequency. I. The Linear Approximation and Geometrical Optics. Phys. Rev. 1968, 166, 1263-1271.

72. Isaacson, R.A. Gravitational Radiation in the Limit of High Frequency. II. Nonlinear Terms and the Effective Stress Tensor. Phys. Rev. 1968, 166, 1272-1279.

(C) 2017 by the authors. Licensee MDPI, Basel, Switzerland. This article is an open access article distributed under the terms and conditions of the Creative Commons Attribution (CC BY) license (http:/ / creativecommons.org/licenses/by/4.0/). 